\title{
A Battle in the Statistics Wars: a simulation-based comparison of Bayesian, Frequentist and Williamsonian methodologies
}

\author{
Mantas Radzvilas ${ }^{1}$ (D) $\cdot$ William Peden ${ }^{2,3}$ (D) Francesco De Pretis $^{1,4}{ }_{\text {(D) }}$
}

Received: 31 October 2020 / Accepted: 31 August 2021 / Published online: 1 November 2021

(c) The Author(s) 2021

\begin{abstract}
The debates between Bayesian, frequentist, and other methodologies of statistics have tended to focus on conceptual justifications, sociological arguments, or mathematical proofs of their long run properties. Both Bayesian statistics and frequentist ("classical") statistics have strong cases on these grounds. In this article, we instead approach the debates in the "Statistics Wars" from a largely unexplored angle: simulations of different methodologies' performance in the short to medium run. We used Big Data methods to conduct a large number of simulations using a straightforward decision problem based around tossing a coin with unknown bias and then placing bets. In this simulation, we programmed four players, inspired by Bayesian statistics, frequentist statistics, Jon Williamson's version of Objective Bayesianism, and a player who simply extrapolates from observed frequencies to general frequencies. The last player served a benchmark function: any worthwhile statistical methodology should at least match the performance of simplistic induction. We focused on the performance of these
\end{abstract}

This article belongs to the topical collection "Recent Issues in Philosophy of Statistics: Evidence, Testing, and Applications", edited by Sorin Bangu, Emiliano Ippoliti, and Marianna Antonutti.

$\bowtie$ William Peden

peden@esphil.eur.nl; w.j.peden@durham.ac.uk

Mantas Radzvilas

mantas.radzvilas@mail.huji.ac.il

Francesco De Pretis

francesco.depretis@unimore.it

1 Department of Biomedical Sciences and Public Health, School of Medicine and Surgery, Marche Polytechnic University, 60126 Ancona, Italy

2 Erasmus Institute for Philosophy and Economics, Erasmus University Rotterdam, 3000 DR, Rotterdam, The Netherlands

3 Centre for Humanities Engaging Science and Society, Durham University, DH1 3HN, Durham, UK

4 Department of Communication and Economics, University of Modena and Reggio Emilia, 42121 Reggio Emilia, Italy 
methodologies in guiding the players towards good decisions. Unlike an earlier simulation study of this type, we found no systematic difference in performance between the Bayesian and frequentist players, provided the Bayesian used a flat prior and the frequentist used a low confidence level. The Williamsonian player was also able to perform well given a low confidence level. However, the frequentist and Williamsonian players performed poorly with high confidence levels, while the Bayesian was surprisingly harmed by biased priors. Our study indicates that all three methodologies should be taken seriously by philosophers and practitioners of statistics.

Keywords Bayesianism · Decision theory · Formal epistemology · Frequentism · Philosophy of statistics · Probability

\section{Introduction}

If there is any suspicion that philosophy of science is an ivory tower subject, it should be extinguished by what Deborah Mayo has called the "Statistics Wars" between classical statisticians, Bayesians, and a prismatic assortment of variations of these views (Ioannidis, 2005; Howson and Urbach, 2006; Wasserstein and Lazar, 2016; Mayo, 2018; van Dongen et al., 2019; Sprenger and Hartmann, 2019; Romero and Sprenger, 2020). Even apparently recondite questions about concepts like evidence, probability, and rational belief are connected with questions of statistical practice. These questions have been given particular salience by the "replication crisis", in which the rates of replication in published statistical research across a range of scientific fields are apparently below what would be expected from random variation alone (Gelman, 2015; Open Science Collaboration, 2015; Smaldino and McElreath, 2016; Fidler and Wilcox, 2018; Trafimow, 2018). All factions within the Statistics Wars make plausible (but often incompatible!) cases that their particular methodology, properly applied, can mitigate some of the malpractices behind the replication crisis. Furthermore, far from being dry debates, the Statistics Wars are frequently characterised by the sort of aggressive rhetoric, bombastic manifestos, and political maneuvering that their name would suggest. And this war-like atmosphere is understandable, because the Wars affect statistical practice, and thereby the health, wealth, and happiness of nations.

Statisticians are often very practical people, so when they are faced with these debates, many of them naturally think that either classical or Bayesian methods can be appropriate in various contexts. Statisticians often say very reasonable things such as "What matters is not who is right about issues in philosophical analysis, but what works best". Some philosophers of science might be attracted to such attempts to pacify the conflicts. Unfortunately, by itself, this pacification strategy fails, because assessing whether a methodology will "work" in a context depends on standards for evaluating what constitutes "working", and as soon as we start determining those standards, we start making choices about the proper analysis of terms like "evidence", "test", "severe test", and for that matter "work".

Yet there is wisdom in the practicing statistician's pragmatism. The philosophical debates within the Statistics Wars are rumbling on with no end in sight, as philosophical debates tend to do. One problems is that the rival statistical methodologies often have 
different goals, e.g. Bayesians often focus more on the decisions of an ideally rational agent, whereas frequentists are often more interested in the reliability of a particular type of test. Statisticians could reasonably use different tools for different contexts. This opens up the opportunity to examine particular types of problem and see which method performs better at achieving some goal that all statisticians share.

One approach to such a question would be historical case studies of real-life cases where one or other method (or both) were used, and then comparing the two. However, it is rarely, if ever, possible to make such comparisons fairly and rigorously. Instead, we employ and expand the use of simulation studies for comparing statistical methodologies. Our simulations use a simple decision problem to pit four players against each other: (1) a confidence interval approach, which classical ("frequentist") statisticians might use for such a problem; (2) conditionalization upon a beta distribution, which many Bayesians would regard as an appropriate family of priors in this context; (3) a hybrid approach based on Jon Williamson's "Objective Bayesian" methodology, which involves forming beliefs about the relative frequencies using confidence intervals and then combines them with an updated version of the Principle of Indifference in order to generate precise probabilities; and (4) a "Sample" player who simply uses the relative frequencies in their observed samples to make point estimates of probabilities, akin to maximum likelihood estimation.

All the methodologies we discuss have some intuitively attractive performance properties as our sample sizes tend towards infinity. Long run performance properties are often given as the raison d'être of frequentist methods, while-in the right sorts of problems and with the right sorts of priors-Bayesian methods will also lead to credences (also called "degrees of belief") that, in the long run, approximate or even match the true relative frequencies (De Finetti, 1980). That is to these theories' credit: we never reach the long run, but information about it arguably provides defeasible information about the short run. If all the methodologies do well in the long run, then the short run is a more promising place to look for divergent performances. For this reason, we used simulations to compare the performance of players inspired by the methodologies when these players were given only small or moderately large samples.

Vituperative rhetoric and dismissive criticisms are common in the Statistics Wars. However, our results indicate that such disrespect is unfounded, at least for the decision problem we study. We found that Bayesianism, frequentism, and (what we call) Williamsonianism can all perform well with suitable player settings. As the number of games becomes large, this similarity can be explained by similar decisions. In the short run, we found differences in decisions. However, with the right player settings, all three of the statistical methodologies can exceed the benchmark that we set for them, and this similarity should increase the respect that the different factions of the Wars have for each other. Our results thus are contrary to what some dogmatic statistical warriors might expect. From each perspective, the other factions might seem absurd, but our study shows how all of them can sometimes lead to good decisions in two senses: (1) the players based on methodologies of statistics do not do significantly worse than the naïve sample-extrapolating player and (2) given the right settings, the players' performances with small samples are not bad in comparison to their performances with more information. 
We briefly review the relevant literature in Sect. 2. We then explain our methods and the "players" in Sect. 3. We display the results and analyse them in Sect. 4, discuss them in Sect. 5, and conclude in Sect. 6.

\section{The Statistics Wars: a multi-dimensional dispute}

The Statistics Wars have a long history, dating back centuries, and they feature a "Who's Who" of statistics. The Wars are sometimes framed in terms of a simple conflict between Bayesian statisticians and classical (or "frequentist") statisticians, but this greatly oversimplifies the debates, as Mayo has detailed (Mayo, 2018). Indeed, no brief summary is possible, but for understanding our simulation study, it helps to note that the participants disagree across multiple dimensions, which we now broadly summarise.

\subsection{The concept of probability}

From a philosopher's perspective, perhaps the most fundamental dimension is which concepts of probability that a statistician regards as appropriate within statistical reasoning. As a preliminary, we must distinguish two potentially divergent domains in which we use probabilistic language: (i) everyday language like "The traffic will probably not be bad today" and (ii) the use of mathematical probability in science, such as a statistic for the probable error of a test. According to some philosophers of probability (including many labelled "frequentist") there is no need for a formal analysis of probabilistic language of type (i). In everyday life, we use "probability" and cognate terms in all sorts of ways, but it is debatable whether there is much of a connection between this ordinary usage and the proper place of probability in scientific reasoning. Insofar as the Statistics Wars are disputes over the proper concept of probability, they are disputes about which concept is appropriate in scientific reasoning.

1a Some theorists adopt physical interpretations of probability, in which probability is variously defined as finite relative frequencies (Venn, 1876), long run hypothetical relative frequencies (von Mises, 1957), propensities (Popper, 1959) or some other type of objective physical magnitude. According to this family of theories, probabilistic statements are generally logically contingent and non-psychological. ${ }^{1}$ Epistemologically, there is no fundamental difference between knowledge of probabilities and other types of knowledge. While probabilities might appear in scientific hypotheses, no hypothesis itself has a probability: it is either true or false, but there is no sense in which statements like "This hypothesis has a probability of 0.5 " can be true with a physical interpretation.

1b Epistemic interpretations analyse probabilistic concepts in terms of an epistemological framework - a system of concepts to do with evidence, belief etc. Within this family, we can distinguish two subgroups. The first subgroup interpret probability psychologically, as either unique (“Objective Bayesian”) (Jaynes, 1957)

\footnotetext{
1 With two obvious exceptions: assertions that are true by definition and assertions within psychology.
} 
or non-unique ("Subjective Bayesian”) (De Finetti, 1980) rational credences. In both cases, probability is analysed as the degree of confidence of either idealized or actual reasoners. The second subgroup of epistemic theorists regard probabilities as "partial entailment" relations between evidential statements and hypotheses (Keynes, 1921; Benenson, 1984; Kyburg, 2001). These "logical" probabilities are arguably guides to rational credences, ${ }^{2}$ but they are not strictly speaking the same thing, just as deductive logical relations (on any non-psychologist philosophy of logic) can often guide rational belief, but cannot be reduced to psychological relations. On either a psychological or partial entailment interpretation, scientific hypotheses can have probabilities.

1c Pluralist combinations of 1a and 1b. For example, Carnap (1945b) combined a partial entailment interpretation of the probabilities of hypotheses (" $H$ 's probability relative to our total evidence is 0.5 " etc.) with a long run relative frequency interpretation of the probabilities mentioned in hypotheses, such as assertions about radioactive half-lives in physics. Pluralistic interpretations are very common among philosophers of science, e.g. Popper (2002), Howson and Urbach (2006), Williamson (2010).

To appreciate these differences in the conceptual analysis of probability, consider how these approaches might interpret some sentences that might occur within scientific reasoning:

$H_{1}$ : "There is a high probability that height will be approximately normally distributed in a large subset of humans."

- Physical: There is a tendency (interpreted in terms of relative frequencies or propensities) of large subsets of humans to be approximately normally distributed with respect to their height.

- Epistemic: On a pure psychological interpretation (such as an uncompromising Subjective Bayesianism) this is an assertion of high confidence that height will be approximately distributed in a large subset of humans, or that a high degree of confidence is rationally appropriate. However, note that most contemporary Subjective Bayesians are pluralists: see below. On a pure partial entailment interpretation, $H_{1}$ is best interpreted as a claim about a probability relation between statements, plus the assertion that one of those statements is true. For instance, we can interpret $H_{1}$ as the combination of three assertions: (i) a statistical hypothesis $H_{S}$, which asserts a physical probability statement such as "There is a high relative frequency of an approximately normal distribution of height among large subsets of human beings", (ii) the claim that $H_{s}$ is our best evidence regarding the single-case hypothesis $H_{t}$ "A particular randomly selected large subset of humans is approximately normally distributed with respect to height", and (iii) a claim that $H_{s}$ has a high partial entailment relation in favour of $H_{t}$ (Benenson, 1984; Kyburg, 1990).

- Pluralist: Most pluralists would adopt a physical interpretation of $H_{1}$, in most contexts.

\footnotetext{
2 Popper (2002) makes use of logical probabilities, without regarding them as mapping onto rational credences; indeed, he entirely rejects the latter concept.
} 
$\mathrm{H}_{2}$ : "It is $99 \%$ probable that there is no life currently on Mars."

- Physical: No relative frequency interpretation of $\mathrm{H}_{2}$ is possible, without being false. $H_{2}$ might have some function in unscientific language as a way of indicating confidence, so a supporter of a relative frequency interpretation might interpret $\mathrm{H}_{2}$ as an informal, extra-scientific expression of confidence. On a propensity interpretation, $\mathrm{H}_{2}$ could be an assertion about the physical chances of life on Mars at this time.

- Epistemic: On a subjectivist psychological interpretation (as in Subjective Bayesianism) $\mathrm{H}_{2}$ is an assertion about the speaker's confidence. On an objectivist psychological interpretation (as in Objective Bayesianism) $\mathrm{H}_{2}$ asserts that $99 \%$ is the degree of confidence, for the hypothesis that there is life on Mars, that is uniquely rational relative to some body of evidence. On a partial entailment interpretation, $\mathrm{H}_{2}$ is an assertion about a partial entailment relation between the hypothesis that there is life on Mars and some body of evidence, such as the total body of evidence in astrobiology.

- Pluralist: Most pluralists would adopt some epistemic interpretation of $\mathrm{H}_{2}$, in most contexts.

\subsection{The evaluation of statistical methods}

If we are testing a statistical hypothesis, then we need to select a method. Often, we might look at what has worked well in the past, but that just pushes the question back: "This method generally works well" is itself a (vague) statistical hypothesis. Additionally, if we are investigating some new or relatively new topic, we might not know what "works". There are three broad families of answers to this question in the Statistics Wars.

2a One criterion is the method's long run performance. For example, suppose that we kept on using a particular procedure for estimating some relative frequency as our sample sizes grew larger and larger. Suppose also that there is a limit that the relative frequency would tend in the long run. ${ }^{3}$ Will the margin of error of our estimates tend towards zero if we stick to this method? If so, does the estimation method have other attractive properties, e.g. does it not depend on the language in which we formulate our hypothesis (Reichenbach, 1938; Feigl, 1954; Salmon, 1967)? Alternatively, we might ask whether the test would almost always provide statistically significant ${ }^{4}$ results in the long run (Fisher, 1947, p. 14)? Alternatively, we may ask whether, in the long run, the test provides a satisfactory ${ }^{5}$ combination of mistaken rejections (Type I errors) and mistaken non-rejections (Type II errors) (Neyman, 1949)?

$2 \mathrm{~b}$ In terms of the doxastic (belief-modifying) properties of the test (Howson and Urbach, 2006; Williamson, 2010). Is the testing procedure a rational way to update

\footnotetext{
3 If there is no limit, then obviously no method will work for estimating it.

4 For some contextually determined standard of statistical significance.

5 As with statistical significance, a "satisfactory" balance must be determined contextually, but Neyman went deep into the decision theory behind such a choice-what he called "inductive behavior" (Neyman, 1957).
} 
our beliefs? Put differently, if we adopt a doxastic approach to evaluation, we assess the test based on how its use would affect the structure of our beliefs. For example, diachronic Dutch Book Arguments are intended to show that, if we adopt some systematic procedure for revising our beliefs that is not an application of Bayesian conditionalization, then we shall be vulnerable to accepting a series of bets in which we will inevitably lose money. Others advocate conditionalization based on its benefits for "epistemic" utilities (Greaves and Wallace, 2006). However, not all philosophers who adopt a doxastic approach to evaluating tests have endorsed conditionalization as a general norm (Bacchus et al., 1990).

2c In terms of whether the testing procedure provides a severe test in a particular context (Mayo, 1996; Spanos, 2010; Mayo, 2018). A "severe" test adequately probes the possible sources of error in our inference, like extrapolations from spurious correlations in the data. In particular, there should be a good chance that our test would uncover a flaw in the hypothesis that we are investigating, if such a flaw exists. A hypothesis might have an excellent fit for our data, but it has not been severely tested unless it was at risk of being rejected by our test, if it were false. Put another way, there should be a low probability that using the testing procedure will lead us to a mistaken inference from the data, i.e. a low "error probability". The role of probability in this methodology is to assess the extent to which tests have probed (or would probe) our hypotheses. Note that severity requires more than just the long term performance properties of $2 \mathrm{a}$ : a test might have good long run properties, but if the test is unlikely to detect a flaw in a single usage and we only evaluate a hypothesis once using that test, then that hypothesis has not been severely tested. In general, according to a severe testing ("error statistical", "probative") evaluation approach, there are problems (such as p-hacking) that do not involve the lack of good long run performance properties, but nonetheless can make our tests inadequate (Mayo, 2018, pp. 13-14).

Naturally, there are interrelations between the criteria used by these three approaches to evaluation. For example, adherents of $2 \mathrm{a}$ and $2 \mathrm{c}$ care about revising our beliefs in rational ways, but evaluate what is a "rational" method for belief revision in terms of long run performance or probative capacity. They believe that the formal models of belief used by adherents of $2 \mathrm{~b}$ are not the right place to start. ${ }^{6}$ Similarly, one intuitive feature of proper doxastic states is that there should be proper coherence between (a) beliefs about long run performance of tests and (b) beliefs in how to update one's beliefs given individual tests. Meanwhile, error statisticians (2c) think that the long run performance qualities of a test are one aspect of its probative quality (Mayo, 2018).

\subsection{Testing procedures and statistics}

Finally, there are testing procedures and test statistics that different methodologists judge to be appropriate in general or in some context. Even given a particular interpretation of probability and shared standards to evaluate our methods, selecting among types of tests can be controversial.

\footnotetext{
6 And, for those methodologists who reject such formal models entirely, not the right place to finish either.
} 
3a Classical tests and test statistics. The former include confidence interval estimation, null-hypothesis significance testing, maximum likelihood estimation, and so on. For our test statistics, we might use p-values, confidence levels, and other descriptive statistics that indicate the long run properties of the testing procedure that we shall implement.

$3 \mathrm{~b}$ Bayesian tests and test statistics, involving the use of conditional probabilities and Bayes' Theorem to update a prior using conditionalization. There are associated test statistics like Bayes factors and posterior probabilities to indicate, respectively, (i) the performance of the tested hypothesis in comparison to another hypothesis and (ii) the warranted degree of belief in the tested hypothesis given the initial prior and the acceptance of the new data.

$3 \mathrm{c}$ Pluralist combinations of $3 \mathrm{a}$ and $3 \mathrm{~b}$. For example, according to Kyburg and Teng (2001), we should use Bayesian methods when we have prior probabilities based on previously inferred statistical information about relative frequencies. However, when such background knowledge is lacking, we should use classical statistics.

Often, in methodological debates, the immediate battleground is one of these dimensions, but disagreements in the others intrude and further complicate the discussions. A simple type of example occurs when, during debates about the adequacy of particular testing procedures, the term "probability" is insufficiently clarified. Researcher A can then say things to Researcher B that are obviously absurd given the other's interpretation of "probability", even though they might be able to develop a practical consensus on the immediate point of disagreement if they made some disambiguations. In such cases, a fundamental disagreement across dimension 1 (Sect. 2.1) unnecessarily frustrates the possibility of consensus on dimension 3 (supra, Sect. 2.3). Moreover, while some of these viewpoints are closely correlated in practice, a great variety of permutations are logically consistent.

Despite the complexities of this debate, we were able to identify three ideas to inspire the three non-benchmark players in our simulations. Thus, in addition to our benchmark player (who naïvely uses their observed sample frequencies) we based on our players on the following methodologies:

A. Frequentism, in the sense of adopting 3a, at least for the reasoning problem that we use in this paper. Thus, we are specifically referring to the methods of classicial statistics and their direct epistemic consequences of providing estimates of relative frequencies. For classical methods, we only need interpretation 1a, although these methods are compatible with other interpretations of probability. In particular, we shall focus on confidence interval estimation. Note that this choice of statistical method for our problem might be justified by any of the evaluative methodologies falling under the categories $2 \mathrm{a}, 2 \mathrm{~b}$, or $2 \mathrm{c}$. For the sake of convenience, we shall assume that a frequentist will also interpret probabilities as relative frequencies, even though this is not necessitated by confidence interval estimation methods.

B. Bayesianism, in the sense of using $3 \mathrm{~b}$ for the problem in our study. Bayesians can adopt either a strictly epistemic interpretation, $1 \mathrm{~b}$, or a pluralist interpretation, 1c. Bayesians more or less invariably arrive at their view via a doxastic approach to evaluating statistical methods, $2 \mathrm{~b}$. 
C. Williamsonianism, by which we mean Jon Williamson's combination of "calibrating" via reasoning that is fundamentally based on classical methods, 3a, but using these relative frequency estimates to guide credences, in accordance with 1b. Given the constraints from beliefs about relative frequencies, the credences are subsequently determined by the Maximum Entropy Principle, described below in Sect. 3.3.3. The overall package is justified by Williamson on doxastic grounds (Williamson, 2010). ${ }^{7}$ In other words, Williamson has an epistemic theory of probability, but there is a large role for classical statistical methods in his theory of inference, leading to divergences from conditionalization (Williamson, 2010, pp. 167-169). Williamsonianism hence offers an interesting combination of ideas that are typically attributed to either frequentism or Bayesianism alone.

Since these methodologies tend to differ across the evaluative dimension, it is extremely difficult to find ways of comparing them that do not beg crucial questions. However, a shared goal is that statistics should help scientists achieve their aims. Scientists have many aims where statistics could reasonably be expected to help: prediction, explanation, control etc. However, one aim of science that seems amenable to formal study is making good decisions under conditions of uncertainty. We mean "decisions" in a broad sense of choices among actions. As Jerzy Neyman pointed out, this is a broad enough definition to include most or all of scientific practice. Decisionmaking includes choosing to publish a paper, to make an expert pronouncement, to invest in developing an experiment, as well as non-scientific decisions like financial investing or trying to design a better mousetrap (Neyman, 1941).

In our simulation, we programmed four different "players" using belief models and choice models. These players were inspired by the three positions in the Statistics Wars that we identified above, plus a benchmark player. The latter enables to see if these methodological positions fulfill a reasonable basic criterion of adequacy: they should do at least as well as someone who just expects future frequencies to match the sample frequency that they have observed thus far. We focused on non-asymptotic decision making, i.e. performance in a finite number of decisions. This might seem to prejudge the case against frequentists, because many of them emphasise long run performance as the proper criterion for testing. However, frequentists are just as interested in making good decisions in the relatively short run as any other other sensible people; that a test procedure has good long run properties is arguably indicative that it will help us to make good decisions in the short run, and frequentists have acknowledged this goal as one objective of statistical inference.

We have no pretense that our study (or simulation studies in general) will or should end the Statistics Wars. Given the persistence of these conflicts throughout the history of statistics, it is probable (in more than one sense) that they are not going to be resolved any time soon. Nonetheless, simulations provide a useful way to assess which methods help guide us towards better decisions and under what conditions. In particular, they can help us evaluate whether frequentism, Bayesianism, or Williamsonianism will enable us to perform systematically better in a particular type of decision problem.

\footnotetext{
7 Williamson uses "Objective Bayesianism" for his view, but we use his name for this approach to distinguish it from (B) and to avoid confusion with the panoply of very different "Objective Bayesianisms".
} 


\section{Methods}

\subsection{Simulations}

Simulations are a relatively new tool in the philosophy of science. For a review up to 2009, see Winsberg (2009). There is an even sparser literature that applies simulations to the Statistics Wars. However, within statistics, there are many studies using simulations to assess Bayesian tools versus frequentist tools for particular problems. To give just a few examples, Avinash Singh et al. compare Bayesian and frequentist methods for estimating parameters of small sub-populations using simulations (Singh et al., 1998). Gilles Celeux et al. use simulations to compare Bayesian regularization methods against frequentist methods (Celeux et al., 2012). Daniel McNeish applies simulations within an investigation of the strengths and weaknesses of Bayesian estimation with small samples (McNeish, 2016). ${ }^{8}$ There is also a literature on Bayesian estimation versus frequentist estimation in structural equation modelling (Smid et al., 2019).

Within philosophy, we found only two earlier studies comparing Bayesian and frequentist methodologies using simulations. Felipe Romero and Jan Sprenger employ simulations to argue in favour of a Bayesian approach to the replication crisis (Romero and Sprenger, 2020). Their study is interesting, but its topic is inferential success under different sorts of scientific institutions, and therefore very different from our own. Instead, our study builds on a philosophically-motivated use of simulations by Kyburg and Teng (1999) to compare frequentist statistics versus Bayesian statistics in a simple decision problem. They use simulations to investigate a game (against Nature) in which a frequentist player and a Bayesian player separately make bets based on a random binomial event - a toss of a coin with unknown bias or fairness. The frequentist player used confidence intervals and the Bayesian player used conditionalization. To serve as a benchmark, Kyburg and Teng also use a player, Sample, who simply estimates that the probabilities will be equal to the frequency in the sum of their samples. After observing some randomly generated coin tosses, players had the option to buy a ticket for heads at a price $t$ or a reversed ticket (effectively betting that the toss will land tails) at a price $(1-t)$. The variable $t$ had a randomly generated price in the interval $[0,1]$. Each player was given a decision algorithm for deciding when and how to bet.

The study then compares the average profits across a range of conditions. The Bayesian player performed at about the benchmark level. Across the full range of possible coin biases that Kyburg and Teng investigated, the Bayesian performed best when their prior corresponded to that required by the Principle of Indifference. ${ }^{9}$ The frequentist tended to make better profits than the Bayesian and the benchmark. Kyburg and Teng do not explain their result, and it has been noted as a puzzle (Schoenfield, 2020, p. 2).

\footnotetext{
8 While we are also interested in small sample inference in this article, we wanted to make comparisons in terms of decision making and also with individual predictions, so McNeish's study was not suitable as a basis for our own research.

9 The Principle of Indifference requires that, if your evidence is equivocal with respect to $m \geq 2$ mutually exclusive and exhaustive states of the world, then your degree of belief in each of them should also be symmetrical: $1 / m$ for each state of the world.
} 
However, comparisons were difficult because the frequentist (unlike the Bayesian) would sometimes refuse to bet. In particular, the frequentist player's algorithm meant that it would not bet if $t$ was within their confidence interval $[x, y]$ where $0 \leq x \leq 1$, $0 \leq y \leq 1$, and $x \leq y$. Kyburg and Teng believed that this feature of the frequentist algorithm meant that this player did much better than the Bayesian in absolute profits. While Kyburg and Teng attempted to address this issue by using average profits rather than total profits, the difficulty remains that the players are being compared over an unequal number of bets. Consequently, it is possible that their results are partly explained by this asymmetry.

Additionally, Kyburg and Teng's study was published in conference proceedings, so quite a few details are obscure, such as whether the players confronted the same sequences of coin tosses and ticket prices. Additionally, they only report the results of 100 simulations for each parameter setting, which means that many of the variations in performance that they find could be explained by random error.

Despite its limitations, the Kyburg and Teng study offers a novel and relatively simple way to compare the decision-theoretic performance of Bayesianism and frequentism in a short run reasoning problem. On the other hand, there was a lot of scope for the expansion, modification, and clarification of their study. Our study design enables comparisons with Kyburg and Teng (1999), but also explores several novel directions; it also attains a level of rigour and detail beyond their conference paper-style investigation. We also sought to reduce the problem of random error by conducting our simulations many times, as we detail in Sect. 3.5. Overall, we conducted two sets of 1000 simulations for each player setting. We also varied the random parametersthe ticket prices and the coin toss results - in the decision problem. Each simulation involved 1000 games, with 1000 simulations for each of five coin biases, and therefore there was a total of 5 million games per player setting in each set of simulations. In this way, by using modern computational power and software, we were able to use a "Big Data" approach that was missing in the study by Kyburg and Teng (1999).

\subsection{Decision problem}

The game that the players faced in our simulations is based around a finite sequence of Bernoulli trials with an initially unknown physical probability. For convenience, we use the terminology of coin tosses, but we stress that, whereas a real-world player would know that a coin is likely to land heads/tails with roughly equal long run frequency (they know that there is a very low proportion very biased coins in the world) in our game they have no such background knowledge about the coin being tossed. However, the players do all know that the tosses are random, in the sense that each toss has an equal (but unknown) chance of landing heads. They also know that the order of coin tosses is irrelevant, i.e. patterns in the sequences of tosses provide no information. Thus, they will only regard sample statistics as relevant evidence for the coin biases.

The basic unit of the decision problem is a decision to bet on heads (action $b_{h}$ ), bet on tails (action $b_{t}$ ), or to hold (action $\bar{b}$ ). Players have known and fixed payoffs for the decisions that we depict in Fig. 1. 
Fig. 1 Player payoff matrix heads tails

\begin{tabular}{|c|c|c|}
\hline$b_{h}$ & $t$ & $-t$ \\
\hline$b_{t}$ & $-(1-t)$ & $(1-t)$ \\
\hline 0 & $-\varepsilon$ & $-\varepsilon$ \\
\hline
\end{tabular}

The variable $t$ is a US dollar (USD) value. It was randomly generated for each game; since it takes values in the $[0,1]$ interval, its average price over the full series of simulations was very probably around 0.5 . Action $b_{h}$ gives a return of $t$ if the result is heads, but incurs a loss $-t$ if the results is tails, because the player bought the ticket at a price $t$ and did not win any money. Action $b_{t}$ gives a return of $(1-t)$ if the result is tails and incurs a loss $-(1-t)$ if the result is heads, again reflecting the price of purchasing the ticket and the absence of a return. Action $\bar{b}$ gives a guaranteed result of $-\varepsilon$. We set $\varepsilon$ to be in the unit interval $[0,1]$.

In Kyburg and Teng (1999), a decision to hold has a guaranteed result of 0. However, as they note (pp. 364-365) it is interesting to see what happens if players are forced to bet. "Force" implies a sanction, and by setting $\varepsilon$ high enough, we can make it rational for a payoff-maximising player to bet. In particular, if $\varepsilon>t$ or $\varepsilon>(1-t)$, then there will always be at least one bet that is rational for the player, because the bet will have a non-negative expected payoff, whereas $-\varepsilon$ is greater than the loss $-t$ or $-(1-t)$. By setting $\varepsilon$ to 1 , we can ensure that all players will bet in each game.

Our simulation presents players with sequences of coin tosses. After a certain number of trials, the players are given a choice whether and how to bet. The players' decisions are completely independent and non-interdependent, so they will not convert to another player's approach if they see that player outperforming them.

\subsection{The players}

Our simulations feature four players, Bayes, Frequentist, Williamsonian, and Sample. The player Sample does not correspond to a standard approach to our decision problem, but it is useful, because a statistical methodology should at least match Sample's performance. Sample can also be interpreted as one way that a frequentist might act if they were forced to give precise relative frequency estimates and use these estimates to guide their decisions. The players have significant differences in their learning rules and decision rules, so we shall discuss them each individually.

\subsubsection{The Bayesian}

Bayes is inspired by Subjective Bayesianism, so there are infinitely many prior probability distributions that they might adopt. However, in our simulations, Bayes will use a 
type of prior that many Subjective Bayesians would use for such a reasoning problem. Bayes knows that the tosses are random. Therefore, they can estimate the probability that a particular toss will land heads simply by deciding one degree of belief in heads for each and every toss. Since they know that each toss will land heads or tails, the probability of tails will be one minus the probability of heads. The beta distribution is a popular option in Bayesian statistics for this type of estimation problem (Mun, 2008, p. 906). The beta distribution enabled us to generate a wide variety of priors for Bayes using just two parameters. For these reasons, we used this procedure for characterising initial priors for Bayes.

Bayes's belief revision procedure in this particular game can be represented with an epistemic model $\mathrm{K}:=\{\Omega, \Theta, H, c, p\}$, where $\Omega:=\{$ heads, tails $\}$ is the set of possible outcomes of a single coin toss (the decision-relevant states), $\Theta:=\left\{\theta \in \mathbb{R}_{\geq 0}: 0 \leq \theta \leq 1\right\}$ is the set of values representing all the possible biases of the coin towards heads (from this point onward, we will simply call $\theta$ "coin bias"), $H:=\{H \cup\{\emptyset\}\}$ is the set of possible histories ${ }^{10}$ with a typical element $h$, where $\mathrm{H}:=\{\text { heads, tails }\}^{n}$ is the set of possible histories that can be generated by $n>0$ coin tosses, such that each $\mathrm{h} \in \mathrm{H}$ is a sequence $\left\{e_{1}, \ldots, e_{n}\right\}$ where each $e_{i} \in\{$ heads, tails $\}, c: H \rightarrow \mathbb{Z}_{\geq 0}$ is a heads event counting function and for every history $h \in H, c(h)=\left|\left\{e_{i} \in h: e_{i}=h e a d s\right\}\right|$, and $p: H \rightarrow \Delta(\Theta)$ is a probability measure which assigns, to every history $h \in H$, a probability distribution on $\Theta$. After observing some history $h \in H$, Bayes revises their beliefs about each possible coin bias $\theta \in \Theta$ via the standard Bayes rule

$$
p(\theta \mid h)=\frac{p(\theta) p(h \mid \theta)}{p(h)}, \text { where } p(\theta)=p(\theta \mid h=\emptyset)>0 \text { denotes a prior. }
$$

Since each coin toss is a Bernoulli trial and the game generates a binomial distribution, we can use a counting function $c$ to reformulate the Bayes rule for each history $h \in H$ as

$$
p(\theta \mid c(h), n)=\frac{p(\theta) p(c(h), n \mid \theta)}{p(c(h), n)},
$$

where $p(c(h), n \mid \theta)=\left(\begin{array}{c}n \\ c(h)\end{array}\right) \theta^{c(h)}(1-\theta)^{n-c(h)}$.

Since our setup ensures that, for every history $h \in H$, the posterior probability distribution $p(\theta \mid c(h), n)$ will be in the same family of probability distributions as prior $p(\theta)$, we can use a conjugate prior and represent the prior by a standard beta distribution with parameters $a$ and $b$. By denoting beta distribution as $\mathrm{B}(a, b)$, we can express the prior as

$$
p(\theta \mid a, b)=\frac{\theta^{a-1}(1-\theta)^{b-1}}{\mathrm{~B}(a, b)}
$$

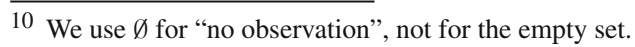


The Bayes rule for a setup with a beta distribution and some history $h \in H$ can be defined as

$$
\begin{aligned}
& p(\theta \mid c(h), n)=\frac{\left(\begin{array}{c}
n \\
c(h)
\end{array}\right) \theta^{c(h)+a-1}(1-\theta)^{n-c(h)+b-1} / \mathrm{B}(a, b)}{\left.\int_{0}^{1}\left(\begin{array}{c}
n \\
c(h)
\end{array}\right) \bar{\theta}^{c(h)+a-1}(1-\bar{\theta})^{n-c(h)+b-1} / \mathrm{B}(a, b)\right) \mathrm{d} \bar{\theta}} \\
& =\frac{\theta^{c(h)+a-1}(1-\theta)^{n-c(h)+b-1}}{\mathrm{~B}(a+c(h), b+n-c(h))} .
\end{aligned}
$$

Notice that this Bayes rule is just another beta distribution with parameters $a+c(h)$ and $b+n-c(h)$. Armed with the prior and the Bayes Rule, Bayes now has all they need both to assign initial priors in the coin toss simulations and to update them in accordance with conditionalization.

Bayes's choices can be represented with a choice model $\mathrm{C}_{\mathrm{b}}:=\{\Omega, \mathrm{D}, H, \mu, \pi\}$, where $\mathrm{D}:=\left\{b_{h}, b_{t}, \bar{b}\right\}$ is the set of possible actions, while $b_{h}$ represents the decision to bet on heads, $b_{t}$ represents the decision to bet on tails, and $\bar{b}$ represents the decision to hold, $\Omega$ represents the outcomes of a single coin toss, $\mu: \Omega \times H \rightarrow X$, where $X:=\left\{x \in \mathbb{R}_{\geq 0}: 0 \leq x \leq 1\right\}$ is the conditional probability function which assigns, to every history $h \in H$, some probability $q \in[0,1]$ on heads and $1-q$ on tails, and $\pi: \mathrm{D} \times \Omega \rightarrow \mathbb{R}$ is the payoff function which assigns, to every possible action-state combination, a real number representing player's payoff. The payoff function assigns payoffs to the action-state combinations in the same way as they are represented in Fig. 1, so that

$$
\begin{array}{ll}
\pi\left(b_{h}, \text { heads }\right)=t ; & \pi\left(b_{h}, \text { tails }\right)=-t ; \\
\pi\left(b_{t}, \text { heads }\right)=-(1-t) ; & \pi\left(b_{t}, \text { tails }\right)=(1-t) ; \\
\pi(\bar{b}, \text { heads })=-\varepsilon ; & \pi(\bar{b}, \text { tails })=-\varepsilon .
\end{array}
$$

The conditional probability function $\mu$ is such that, for history $h=\emptyset$, the probability of heads is

$$
\mu(\text { head } s \mid h=\emptyset)=\int_{0}^{1}(p(\theta) \theta) \mathrm{d} \theta,
$$

while $\mu$ (tails $\mid h=\emptyset)=1-\mu($ heads $\mid h=\emptyset)$.

For every history $h \in \mathrm{H}$ the probability of heads is

$$
\mu(\text { heads } \mid h)=\int_{0}^{1}(p(\theta \mid c(h), n) \theta) \mathrm{d} \theta,
$$

while $\mu($ tails $\mid h)=1-\mu($ heads $\mid h)$.

The expected payoff from some action $\mathrm{d} \in \mathrm{D}$ given some history $h \in H$ is

$$
\mathbb{E}_{\pi}[\mathrm{d} \mid h]=\pi(\mathrm{d}, \text { heads }) \mu(\text { heads } \mid h)+\pi(\mathrm{d}, \text { tails }) \mu(\text { tails } \mid h) .
$$


We assume that Bayes is a rational player who seeks to maximise the expected payoff. Thus, for any history $h \in H$, Bayes always chooses an action $\mathrm{d} \in \mathrm{D}$, such that

$$
\mathbb{E}_{\pi}[\mathrm{d} \mid h] \geq \mathbb{E}_{\pi}[\overline{\mathrm{d}} \mid h], \text { for all } \overline{\mathrm{d}} \in \mathrm{D}
$$

When more than one action satisfies this requirement, Bayes will make a random choice among these actions. Since hold is never a strictly dominant option, we simplified Bayes by supposing that they never choose this option.

\subsubsection{The Frequentist}

Just as Subjective Bayesians might adopt many different priors for a coin tossing problem, so there are multiple ways that a frequentist might estimate the relative frequencies involved, i.e. the frequencies of heads and tails among the tosses. However, many frequentists would regard estimation using confidence intervals as a reasonable method for our decision problem, in which Frequentist starts out with no initial estimate of what the relative frequency might be and where they want to estimate the general relative frequency for the purpose of guiding their betting behaviour on particular tosses. Additionally, it is this approach that Kyburg and Teng, who are frequentists (in the broad sense that we are using in this article) adopt for their frequentist player.

We explain the technical details of how Frequentist works below, but we shall also provide an informal summary beforehand. Frequentist will estimate that, in the population of coin tosses, the relative frequency of heads is within a confidence interval. Since they know that all the tosses will land heads or tails, that confidence interval also provides them with an estimate of the relative frequency of tails. A "confidence interval" is an estimate that a population frequency is within some range. Let us focus on the long run relative frequency of heads in the coin tosses. This is the relative frequency that a hypothetical infinite series of tosses would approach in the limit as the number of tosses increased. A confidence interval of $[0.4,0.6]$ for the long run frequency of heads in the population of coin tosses is an estimate that the relative frequency of the coin landing heads is at least $40 \%$ of the coin tosses and no more than $60 \%$ of them. Frequentist will tentatively believe that the relative frequency for the coin toss population is somewhere in the estimated interval. They will tentatively dismiss values outside this range, such as 0.2 or 0.9 , as possible values for the relative frequency. Frequentist's beliefs are "tentative" in the sense that, with more sample data, they can change the interval to very different values; a confidence interval using one sample for estimation could be $[0,0.5]$, but the interval using an enlarged sample could be $[0.9,0.99]$.

The confidence level is not a probability, but instead a value describing the procedure used to estimate the intervals. We stipulate that Frequentist knows that the sampling assumptions for confidence interval testing are satisfied. The confidence level is set via a parameter $\alpha$, between 0 and 0.5 , such that the level is $(1-\alpha)$. The value of $\alpha$ is exogenous to the confidence interval methodology, so we simulated how Frequentist will perform with a range of values of $\alpha$. The confidence level tells us, for a particular sample size, the minimum possible long run relative frequency of correct estimates of 
a confidence interval of that width using that sample size. For example, if $\alpha=0.05$, then the confidence level for the intervals estimated using this method is 0.95 , or $95 \%$. The level is only the minimum, because if the population is homogeneous-all tosses land heads or all tosses land tails - then the success rate will be 100\%. Therefore, the success rate in the example could be anywhere from 95 to $100 \%$. Once Frequentist has estimated a confidence interval given their existing observations, they will use it to guide their betting behaviour for the individual tosses on which they can bet. ${ }^{11}$

We now formulate this belief revision procedure in detail. Frequentist revises their estimate of the coin bias in a way that can be represented with a model $\mathrm{F}:=\left\{\Omega, \mathrm{H}, \mathrm{k}_{\alpha}, \mathrm{c}\right\}$, where $\Omega$ is the set of possible outcomes of a single coin toss, $\mathrm{H}=\{\text { heads, tails }\}^{n}$ is the set of possible histories which can be generated with $n>0$ coin tosses, ${ }^{12} \mathrm{c}: \mathrm{H} \rightarrow \mathbb{Z}_{\geq 0}$ is the heads event counting function and for every history $\mathrm{h} \in \mathrm{H}, \mathrm{c}(\mathrm{h})=\mid\left\{e_{i} \in \mathrm{h}: e_{i}=\right.$ heads $\} \mid$, and $\mathrm{k}_{\alpha}: \mathrm{H} \rightarrow \mathrm{P}(X)$, where $X:=\left\{x \in \mathbb{R}_{\geq 0}: 0 \leq x \leq 1\right\}$, is a function which assigns, to every history $\mathrm{h} \in \mathrm{H}$, an interval $\mathrm{k}_{\alpha}(\mathrm{h})=\left(k_{l}, k_{u}\right)$ with lower bound $k_{l}$ and upper bound $k_{u} \geq k_{l}$. Since the game is a sequence of Bernoulli trials that generates a binomial distribution, we can determine Frequentist's confidence interval by calculating the Clopper-Pearson interval. For any history $\mathrm{h} \in \mathrm{H}$, the lower and upper boundaries of the Clopper-Pearson interval can be represented as the following beta distribution quantiles

$$
\begin{aligned}
& k_{l}=\mathrm{B}\left(\frac{\alpha}{2} ; \mathrm{c}(\mathrm{h}), n-\mathrm{c}(\mathrm{h})+1\right) \\
& k_{u}=\mathrm{B}\left(1-\frac{\alpha}{2} ; \mathrm{c}(\mathrm{h})+1, n-\mathrm{c}(\mathrm{h})\right) .
\end{aligned}
$$

Thus, after observing a history of $n$ coin tosses $\mathrm{h} \in \mathrm{H}$ with $\mathrm{c}(\mathrm{h}) \geq 0$ heads, if Frequentist adopts a an level $1-\alpha$, they will estimate the actual coin bias to be within an interval $\mathrm{k}_{\alpha}(\mathrm{h})=\left(\mathrm{B}\left(\frac{\alpha}{2} ; \mathrm{c}(\mathrm{h}), n-\mathrm{c}(\mathrm{h})+1\right), \mathrm{B}\left(1-\frac{\alpha}{2}\right.\right.$; c (h) $+1, n$ $-\mathrm{c}(\mathrm{h})))$, and they will not take into account any value $k \notin \mathrm{k}_{\alpha}(\mathrm{h})$.

There is no consensus on how to model decisions with interval-valued beliefs. There are many options in the literature (Resnik, 1987). However, we programmed Frequentist to use what seems to be the decision procedure in Kyburg and Teng (Kyburg and Teng, 1999), which we also interpreted using other publications by Kyburg, in particular (Kyburg, 1990, 2003).

Frequentist's choices can be represented with a choice model $\mathrm{C}_{\mathrm{f}}:=\left\{\Omega, \mathrm{D}, \mathrm{H}, \mathrm{k}_{\alpha}, \pi\right.$, $\phi$, where $\Omega$ is the set of possible outcomes of a coin toss, D is the set of possible actions, $\mathrm{H}$ is the set of possible histories, $\mathrm{k}_{\alpha}$ is the function which assigns a Clopper-Pearson interval to every history $h \in H, \pi$ is a payoff function identical to the one defined for Bayes player, and $\phi: \mathrm{D} \times \mathrm{H} \rightarrow \mathrm{P}(\mathbb{R})$ is a function

\footnotetext{
11 In our decision problem, where the players all know that the coin tosses are random, we can safely assume that Frequentist should use the estimates of the general relative frequency to form their beliefs about particular tosses. In problems where Frequentist is confronted with e.g. different statistics for different overlapping reference classes, the applicability of this assumption becomes much more complicated, as frequentists have known since at least (Venn, 1876). For influential discussions, see also Salmon (1967) and Reichenbach (1971).

12 The method used to compute the confidence interval cannot be employed when $h=\emptyset$. However, the first bet in the game occurs only after each player observes a number of coin tosses.
} 
which assigns, to every action-history pair $(\mathrm{d}, \mathrm{h}) \in \mathrm{D} \times \mathrm{H}$, an expected payoff vector $\phi(\mathrm{d}, \mathrm{h})=\left(\mathbb{E}_{\pi}[\mathrm{d}, \underline{k}], \ldots, \mathbb{E}_{\pi}[\mathrm{d}, \bar{k}]\right)$, where $\underline{k}=\min \left(\mathrm{k}_{\alpha}(\mathrm{h})\right), \bar{k}=\max \left(\mathrm{k}_{\alpha}(\mathrm{h})\right)$, and $\mathbb{E}_{\pi}[\mathrm{d}, k]=k \pi(\mathrm{d}$, heads $)+(1-k) \pi(\mathrm{d}$, tails $)$ for every $k \in \mathrm{k}_{\alpha}(\mathrm{h})$.

The choice behaviour of Frequentist can be defined with the interval-dominance principle. Action $\mathrm{d} \in \mathrm{D}$ interval-dominates action $\overline{\mathrm{d}} \in \mathrm{D}$ if and only if, given history $\mathrm{h} \in \mathrm{H}, \min (\phi(\mathrm{d}, \mathrm{h}))>\max (\phi(\overline{\mathrm{d}}, \mathrm{h}))$. For any pair of actions $\mathrm{d} \in \mathrm{D}$ and $\overline{\mathrm{d}} \in \mathrm{D}$, Frequentist always chooses action $\mathrm{d}$ if $\mathrm{d}$ interval-dominates action $\overline{\mathrm{d}}$, and is indifferent between $\mathrm{d}$ and $\overline{\mathrm{d}}$ when neither action interval-dominates the other. Reasoning this way almost corresponds extensionally to the frequentist player's behaviour in Kyburg and Teng (1999), where no general decision rule is provided. ${ }^{13}$

We now apply this approach to the coin tossing problem. We define $\triangleright$ as denoting the interval-dominance of one action over another and $\circ$ for when neither action intervaldominates the other. For a particular confidence interval and the fixed utilities given in Fig. 1, the actions $b_{h}, b_{t}$, and $\bar{b}$ can stand in the following relations:

(i) $b_{h} \triangleright b_{t}$ and $b_{h} \triangleright \bar{b}$;

(ii) $b_{t} \triangleright b_{h}$ and $b_{t} \triangleright \bar{b}$;

(iii) $\bar{b} \triangleright b_{h}$ and $\bar{b} \triangleright b_{t}$;

(iv) $b_{h} \triangleright b_{t}$ and $b_{h} \circ \bar{b}$;

(v) $b_{h} \circ b_{t}$ and $b_{h} \triangleright \bar{b}$;

(vi) $b_{t} \triangleright b_{h}$ and $b_{t} \circ \bar{b}$;

(vii) $b_{h} \circ b_{t}$ and $b_{h} \circ \bar{b}$.

Frequentist will choose $b_{h}$ in case (i), $b_{t}$ in case (ii), and $\bar{b}$ in case (iii). They will make random choices between $b_{h}$ and $\bar{b}$ in case (iv), $b_{h}$ and $b_{t}$ in case (v), $b_{t}$ and $\bar{b}$ in case (vi), and between all three actions in case (vii). Our decision algorithm for Frequentist in the code embodies this behaviour. ${ }^{14}$

\subsubsection{The Williamsonian}

Williamsonian is somewhat of a hybrid player. Consequently, after our discussion of the previous two players, there are few new notions involved in their reasoning. Williamson (2010) argues for three fundamental principles about probabilistic reasoning:

\footnotetext{
13 However, in Kyburg and Teng (1999), if no action interval-dominates holding, their frequentist player will always choose to hold. Kyburg and Teng provide no justification for this feature of their frequentist player's decision algorithm, so we have interpreted it as a programming simplification, and one that was not helpful for our simulations.

14 One might object that it is unfair to enable Frequentist to hold, while Bayes never makes this decision. This modelling choice has the advantage that our simulations are more comparable to those of Kyburg and Teng (1999). It is also acceptable from Bayes's perspective, since hold is never strictly dominantat best, Bayes is indifferent between this option and some other option. However, an anonymous referee points out that less conventional Bayesian reasoners will not necessarily share this perspective. In particular, "imprecise" Bayesians, who model agents using sets of probability functions, do not identify the expected value of not taking a bet to be the negative of the bet's expected value. In their model, the minimum price at which an agent is willing to sell a ticket and the minimum price at which they are willing to buy that ticket can differ, in a similar way to Frequentist. For some influential discussions and excellent expositions, see Levi (1974), Gilboa and Schmeidler (1989), Seidenfeld (2004), Troffaes (2007), Bradley (2017). We look forward to investigating the performance of such players in future research. In the present study, we address this issue using $\epsilon=1$, which enables us to simulate what happens when we remove hold as an acceptable option for Frequentist.
} 
1. Probabilism: Beliefs should be representable as credences satisfying the additive probability calculus.

2. Calibration: These credences should reflect any relevant knowledge about relative frequencies.

3. Equivocation: Subject to the constraints from Calibration, the credences should be maximally equivocal among the different possible states of the world.

The principle of Probabilism is a familiar Bayesian idea. For Calibration, Williamson endorses Kyburg's system of "Evidential Probability" (Wheeler and Williamson, 2011) and this system requires using confidence intervals where (as in our simulations) there is no background knowledge of the relevant conditional probabilities for events (Kyburg and Teng, 2001, p. 264). Consequently, Williamsonian will estimate confidence intervals in the same way as Frequentist.

However, Equivocation and Probabilism entail that Williamsonian will differ from Frequentist in the other parts of their learning procedure. To determine equivocal credences in a systematic manner, Williamsonian uses the "maximum entropy principle". Williamson gives the full formal connections between entropy maximisation and formal beliefs in Williamson (2010), building on research such as Jaynes (1957). The salient point is that, given a confidence inferred at some exogenously determined confidence level $(1-\alpha)$ and a set of $m \geq 2$ exhaustive and mutually exclusive states of the world that are consistent with the relative frequencies estimated using that confidence level, a Williamsonian will try to minimise the distance between their probabilities for each state of the world and the value $1 / \mathrm{m}$ implied by the Principle of Indifference.

Williamsonian's belief revision can be represented with a model $\mathrm{W}:=\{\Omega, \Theta, \mathrm{H}, \mathrm{p}$, $\left.\mathrm{k}_{\alpha}, \mathrm{c}, p_{w}\right\}$, where $\Omega$ is the set of possible outcomes of a single coin toss, $\Theta$ is the set of values representing all the possible coin biases, $\mathrm{H}$ is the set of possible histories that can be generated by $n>0$ coin tosses, $\mathrm{c}$ is the heads event counting function, $\mathrm{p}: \Theta \rightarrow \Delta(\Theta)$ is a function which assigns a uniform probability distribution on $\Theta$, $\mathrm{k}_{\alpha}$ is a function which assigns a Clopper-Pearson interval to every history $\mathrm{h} \in \mathrm{H}$, and $p_{w}: \mathrm{H} \rightarrow X$, where $X:=\left\{x \in \mathbb{R}_{\geq 0}: 0 \leq x \leq 1\right\}$, is a Williamsonian belief function which assigns, to every history $\mathrm{h} \in \mathrm{H}$, a belief $p_{w}(\mathrm{~h})$, such that

$$
p_{w}(\mathrm{~h}) \in \operatorname{argmin}_{k \in \mathrm{k}_{\alpha}(\mathrm{h})}\left|k-\int_{0}^{1}(\mathrm{p}(\theta) \theta) \mathrm{d} \theta\right| .
$$

Since function $\mathrm{p}$ assigns a uniform distribution on $\Theta$, we have a case where $\int_{0}^{1}(\mathrm{p}(\theta) \theta) \mathrm{d} \theta=1 / 2$. Thus, we can rewrite condition 11 as

$$
p_{w}(\mathrm{~h}) \in \operatorname{argmin}_{k \in \mathrm{k}_{\alpha}(\mathrm{h})}\left|k-\frac{1}{2}\right| .
$$

Therefore, in our coin tossing problem, Williamsonian first estimates confidence intervals for heads and tails in general at a confidence level $(1-\alpha)$. Secondly, subject to this constraint, they set a Bayesian probability. For example, suppose that the Williamsonian has estimated a confidence interval $[0.4,0.6]$ for heads. This is consistent with assigning a probability of 0.5 for each particular toss landing heads, so 
Williamsonian makes 0.5 their degree of belief. However, if the interval is [0.1, 0.4], then 0.5 is not consistent with their beliefs about the relative frequencies. Instead, 0.4 is the available value that maximises entropy, and hence Williamsonian makes 0.4 their degree of belief in each particular toss landing heads.

Williamsonian's choices can be characterised with choice model $\mathrm{C}_{\mathrm{w}}:=\{\Omega, \mathrm{H}, \mathrm{D}, \pi$, $p_{w}$ \}, where $\Omega$ is the set of coin toss outcomes, $\mathrm{H}$ is the set of histories, $\mathrm{D}$ is the set of actions, $\pi$ is the payoff function identical to that which we defined for Bayes and Frequentist players, and $p_{w}$ is a Williamsonian belief function. Williamsonian's expected payoff associated with some action $d \in D$ given some history $h \in H$ is

$$
\mathbb{E}_{\pi}[\mathrm{d} \mid \mathrm{h}]=p_{w}(\mathrm{~h}) \pi(\mathrm{d}, \text { heads })+\left(1-p_{w}(\mathrm{~h})\right) \pi(\mathrm{d}, \text { tails }) .
$$

We assume that Williamsonian is an expected payoff maximizer and thus, for any history $\mathrm{h} \in \mathrm{H}$, always chooses action $\mathrm{d} \in \mathrm{D}$, such that $\mathbb{E}_{\pi}[\mathrm{d} \mid \mathrm{h}] \geq \mathbb{E}_{\pi}[\overline{\mathrm{d}} \mid \mathrm{h}]$, for all $\overline{\mathrm{d}} \in \mathrm{D}$.

Despite the similarities in the learning procedures for Williamsonian and Frequentist, the principle of Equivocation leaves Williamsonian in a very different place, because the latter has a precise probability when they face each next coin toss. Therefore, Williamsonian makes decisions, for a particular set of credences, in an identical way to Bayes.

\subsubsection{Sample}

Our final player, Sample, serves two primary functions. Firstly, they can be interpreted as Frequentist in a situation where they are forced to make precise estimates of the relative frequencies of heads. In that case, Frequentist might estimate the general relative frequencies of heads to be the limit as $\alpha \rightarrow 0$, which is just the sample frequency. Thus, Sample may be interpreted as estimating that the long run frequency of heads is just the relative frequency in the total sample that they have observed so far. These estimates then guide Sample's bets on each given toss. Sample also thereby has estimates for tails, given their knowledge of the coin tossing set-up. Sample's second function is as a benchmark: if a statistical methodology's performance is inferior to just estimating a precise probability using the sample frequency, then this is a bad mark against that methodology.

Conceptually, there is nothing novel in Sample's belief revision procedure. They can be interpreted in multiple ways: as a version of Frequentist with precise estimates of relative frequencies, as a quasi-Bayesian reasoner (similar to the " $\lambda=0$ " agent in Carnap, 1952) and so on. The divergence with Frequentist is mainly in how different sample sizes are treated: given a 0.5 sample frequency of heads and a sample of 4, Sample is certain that the limiting relative frequency of heads in the tosses is 0.5 , whereas Frequentist has a very wide confidence interval around 0.5; given a 0.5 sample frequency of heads and a sample size of 1000, Sample's beliefs are the same as when they had just 4 observations, whereas Frequentist has a very narrow confidence interval. Even this divergence can be reduced if we interpret Sample as a frequentist reasoner who uses the sample frequency as a decision-making tool, without interpreting the concomitant probability distributions as degrees of belief. This type of reasoner is 
briefly discussed by Williamson (2007). The divergence with Bayesianism is that Sample has no initial priors, and thus does not update by conditionalization. While Sample is not a true Bayesian reasoner, in our simulations they will always have some sample data prior to making decisions, and therefore there is no need to specify the beliefs of Sample when they lack sample data.

For the choice behaviour of Sample, we model them as possessing a precise probability (interpreted either as a relative frequency estimate or as a non-Bayesian credence) for each possible outcome of each toss. Given this probability, it is natural for Sample to use Bayesian decision theory, because even many frequentists would say that such choices would be appropriate if one legitimately possessed the relevant relative frequency estimates, and Sample views themselves as having this information. Thus, for given credences, Sample makes decisions in the same way as Bayes. This common decision algorithm has the added advantage that Sample only differs from Bayes in their learning methods, which helps comparisons between the two players.

\subsection{Coding and simulation architecture}

Starting from the common decision problem and particular settings specified for each player in Sects. 3.2 and 3.3, we coded six different Python 3 scripts (version 3.8.1), based on the statsmodel econometric and statistical library (Seabold and Perktold, 2010) to perform the simulations. The first two scripts generated coin tosses and ticket prices. This data was subsequently employed as an input for the other four scripts. The output of the latter scripts gave us the cumulative monetary mean profits for each player, like those shown below in Tables 1, 2, 3, 4, 5 and 6 and, focusing on the Frequentist, the statistics of the conditions met by this player (see end of Sect. 3.3.2) in the simulations, as outlined in Tables 7 and 8. All simulations were performed on an Ubuntu Linux server powered by an 8 cores (16 threads) Intel Xeon (Skylake type) processor@2.2 GHz. High performance data multiprocessing meant that the overall computational time was approximately 1.5 hours. The simulation results were stored in 310,000 different txt files for about 3.5 gigabytes. The code is available from the authors upon request.

\subsection{Variations}

The games each consisted of a number of observations of coin tosses, which were used by the players to update their initial belief state. Once these beliefs were updated, players used their decision algorithms to choose an action. Players retained information from game-to-game within a particular simulation, but they did not retain any information from one simulation to another. 


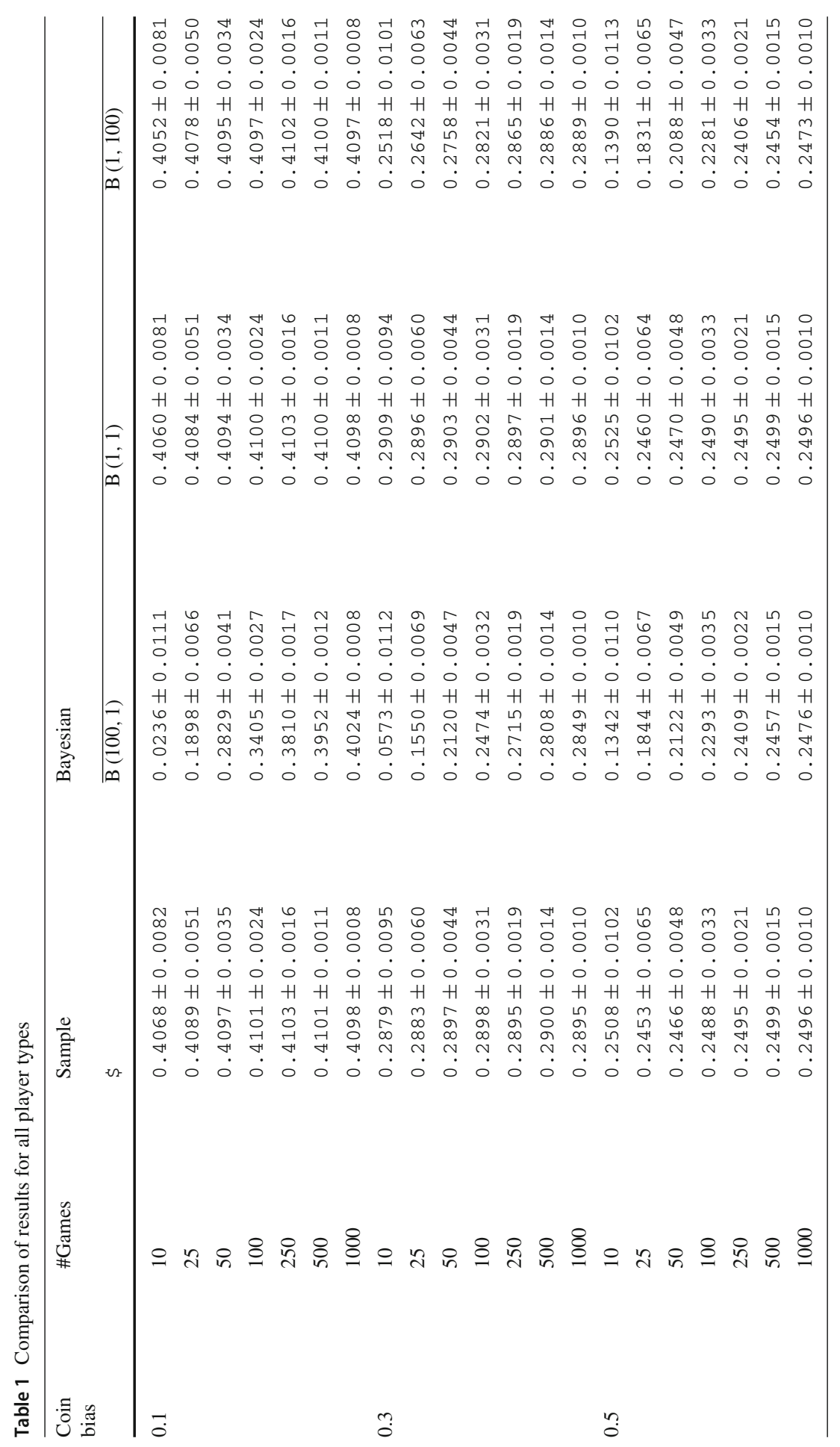




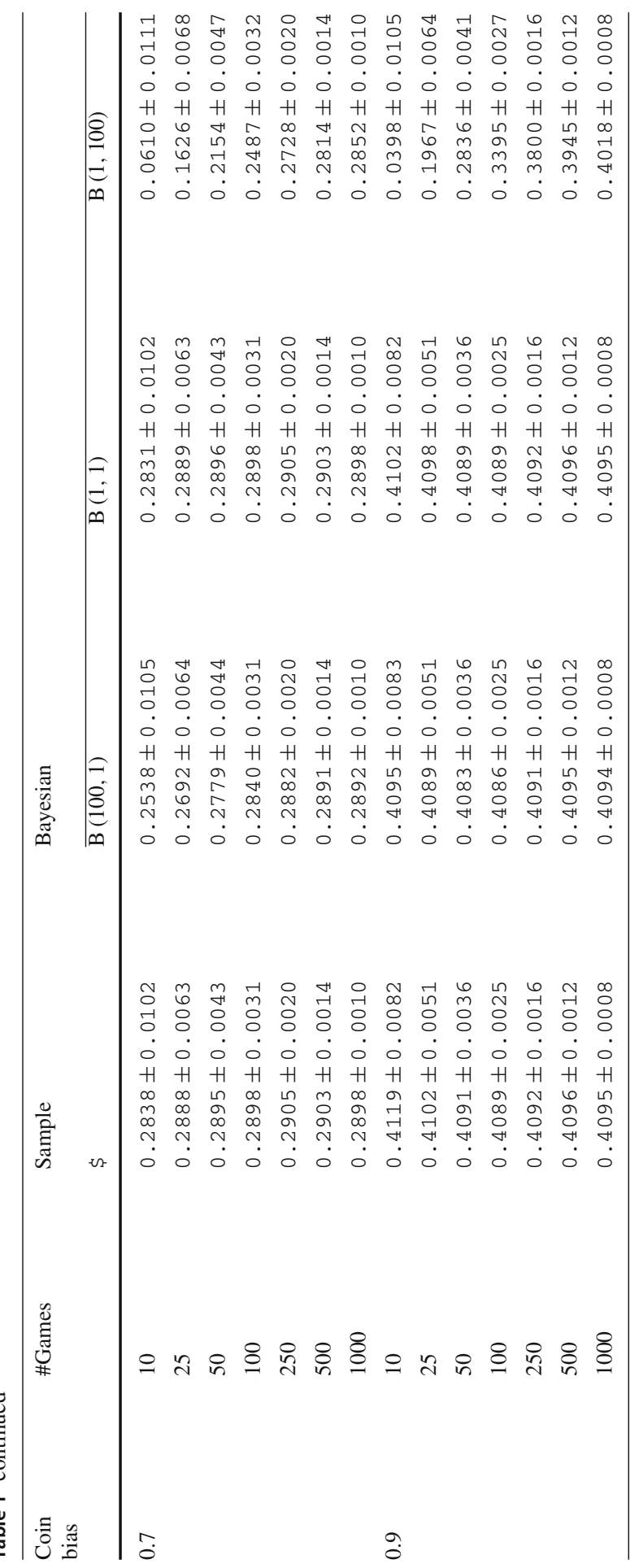




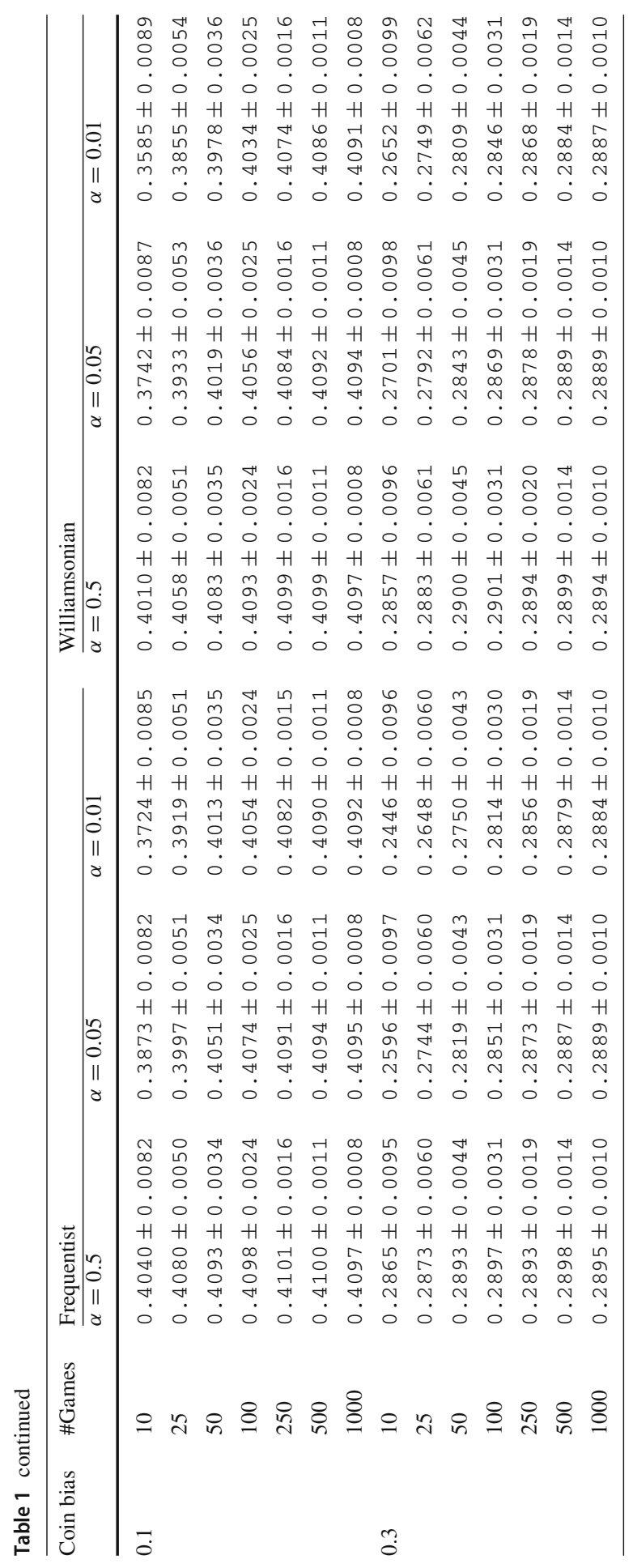




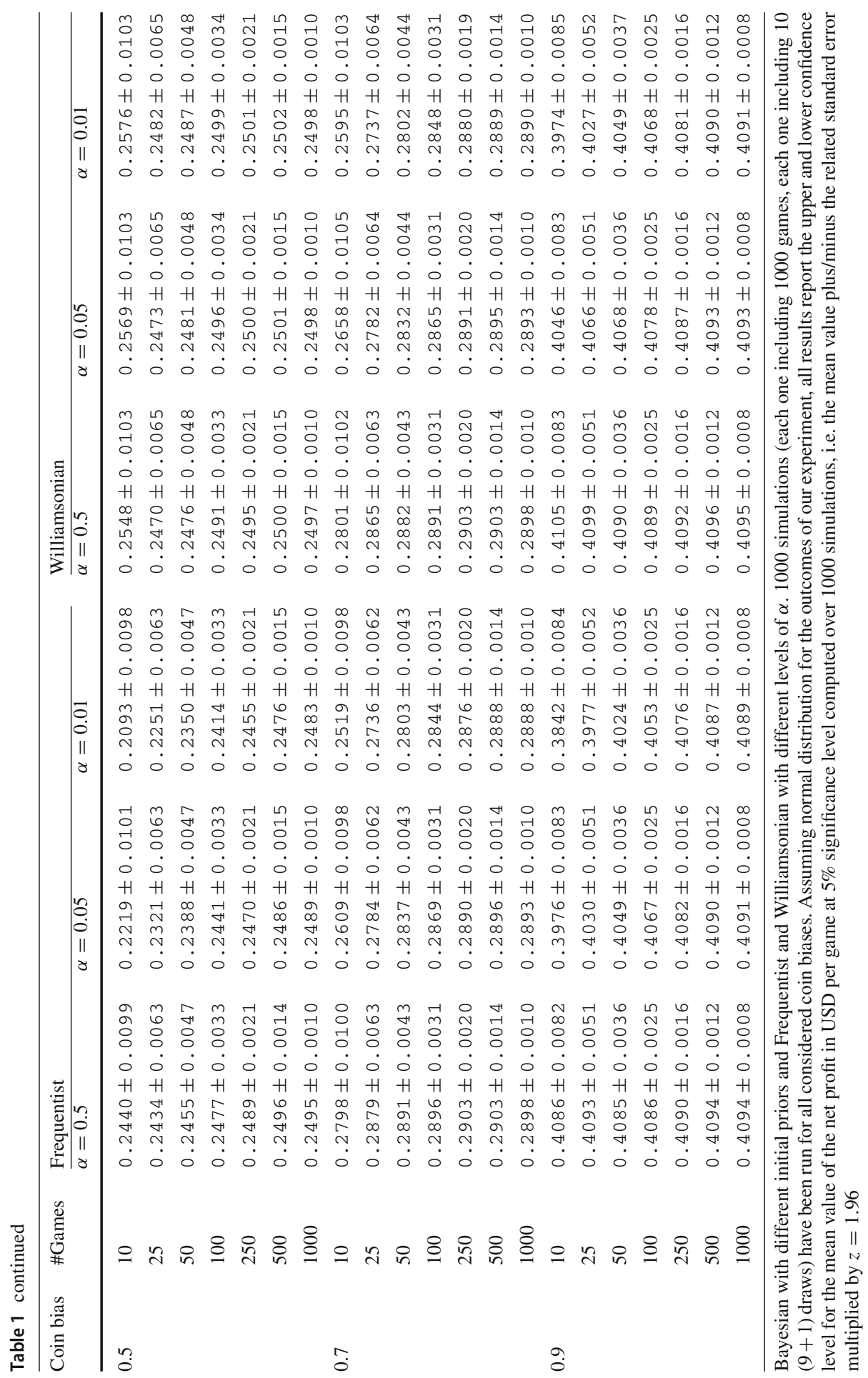




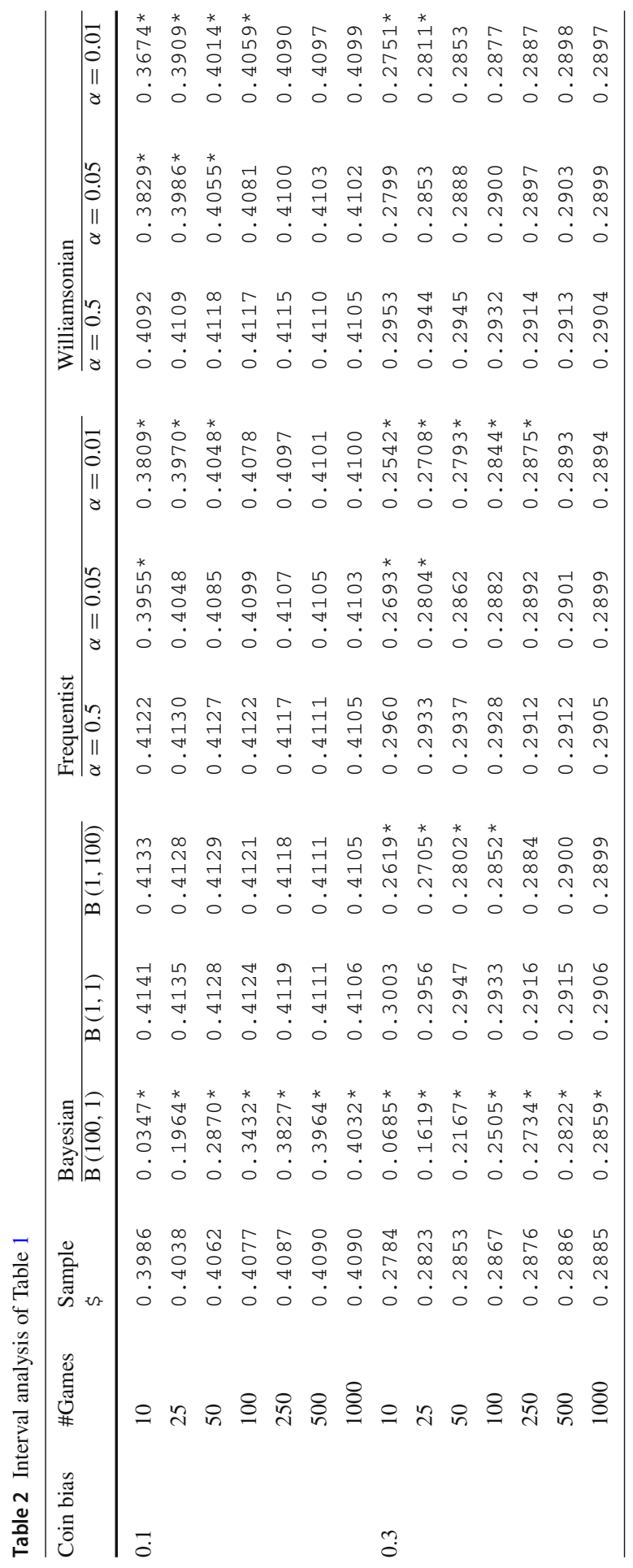




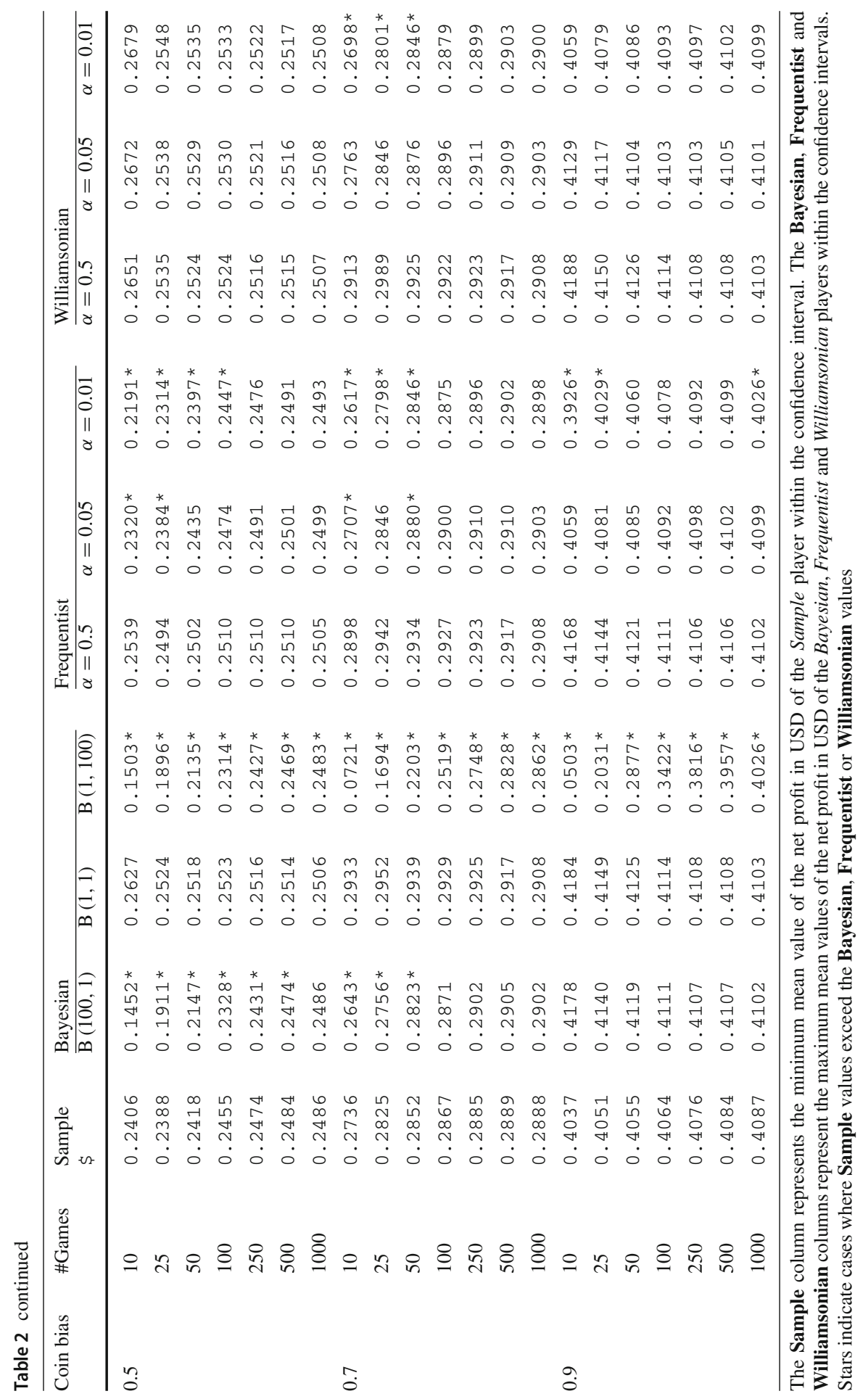




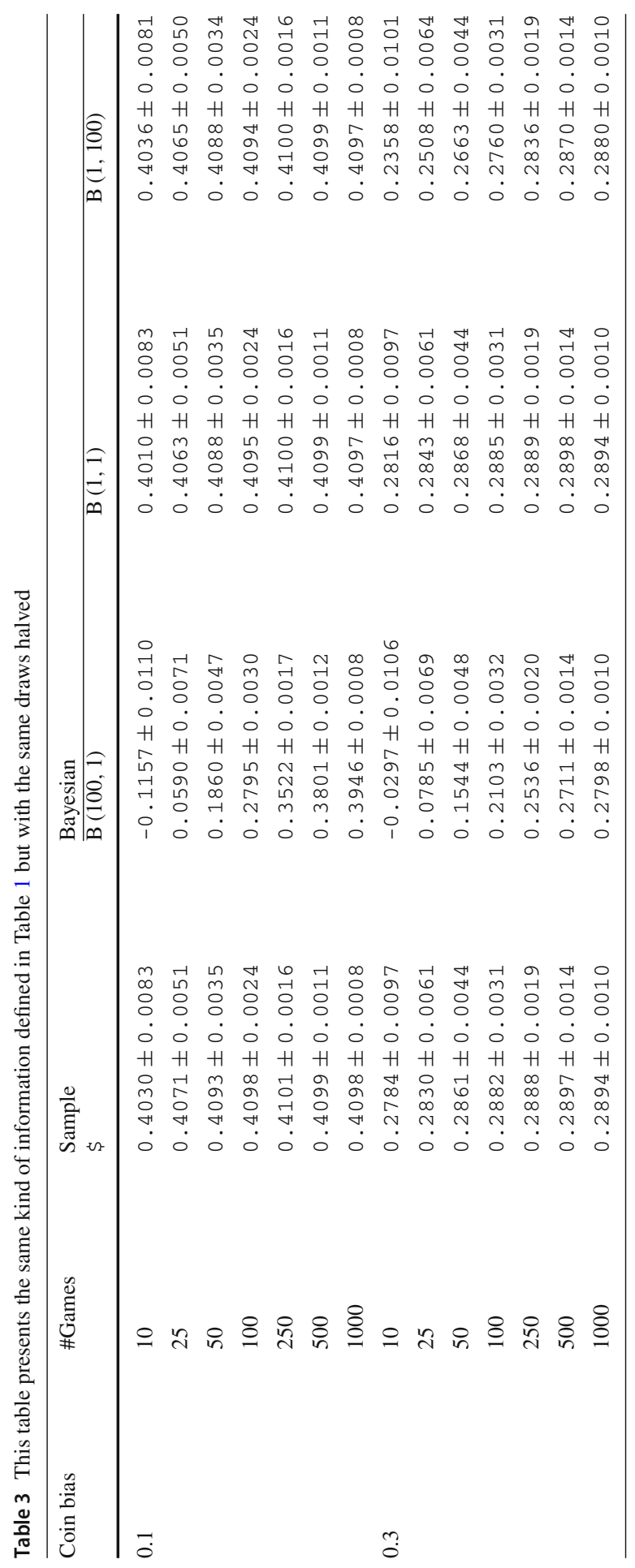




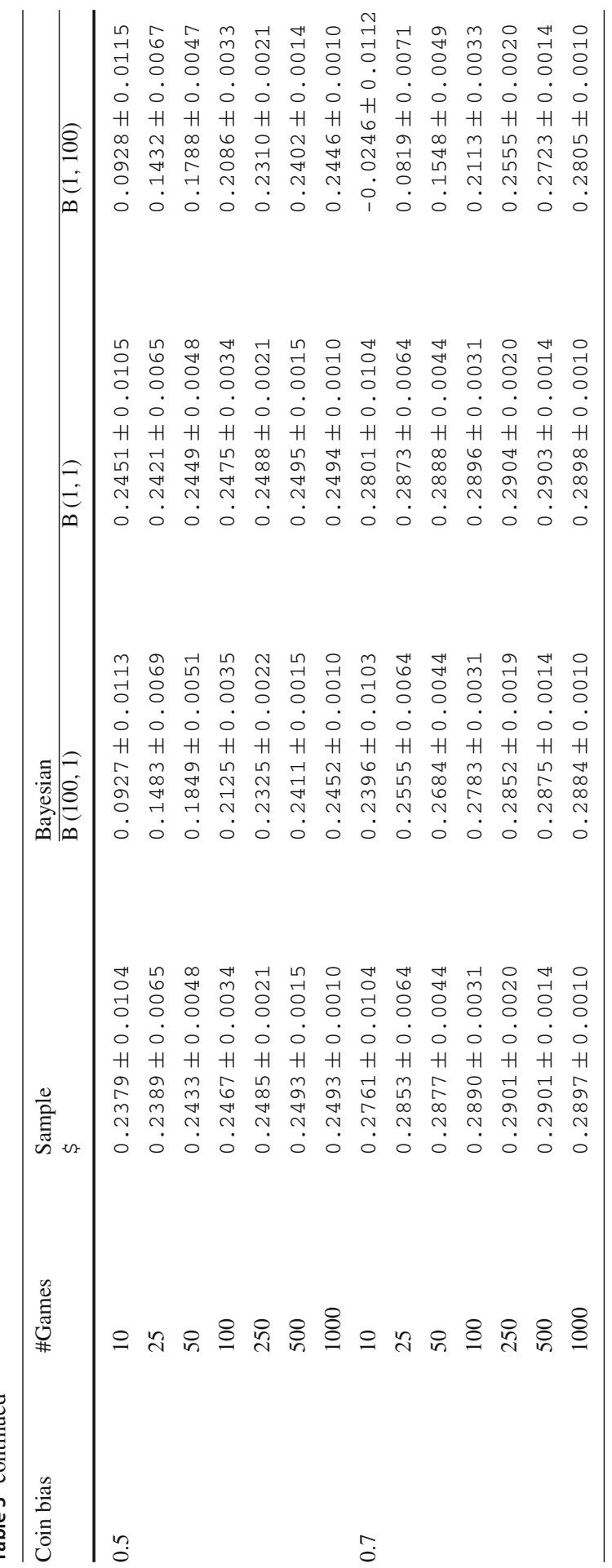




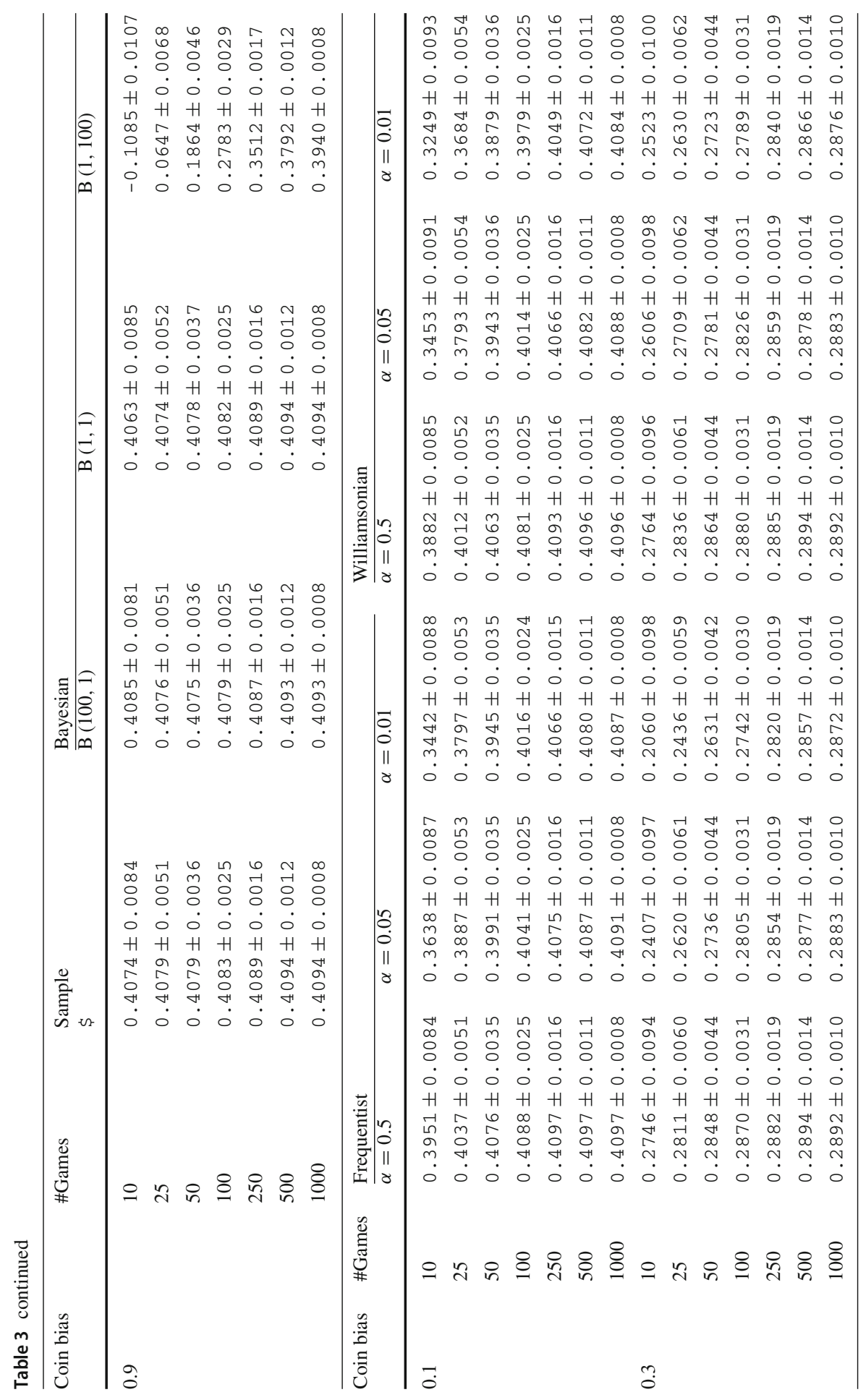




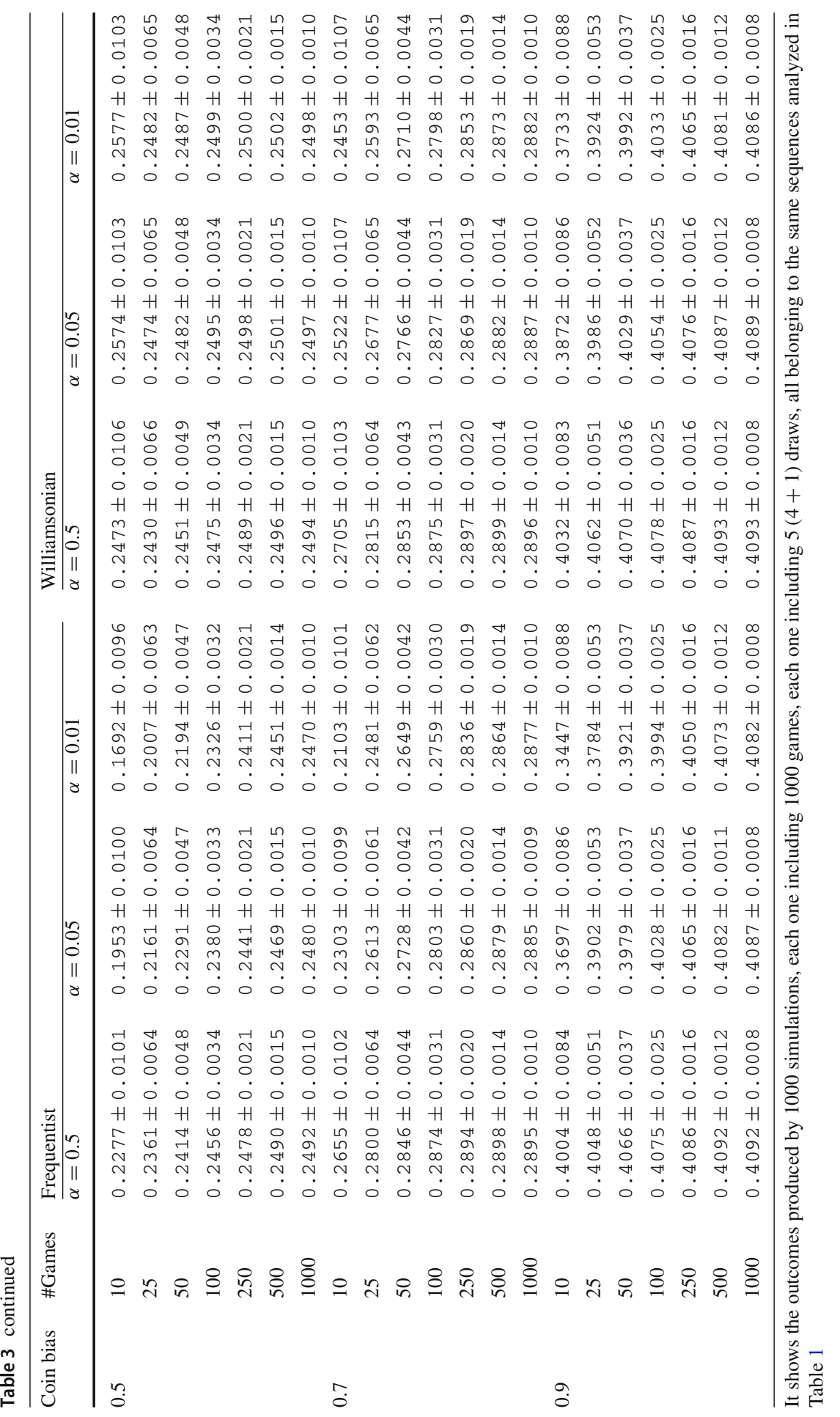




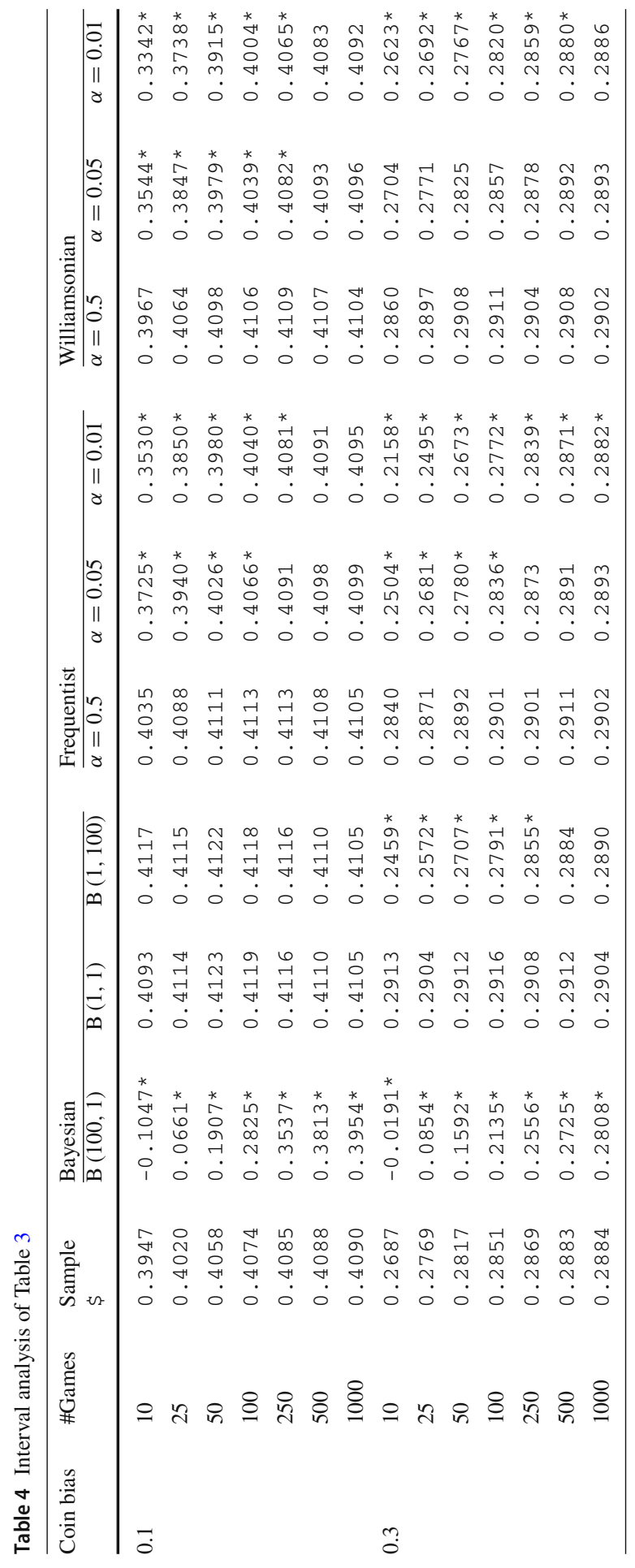




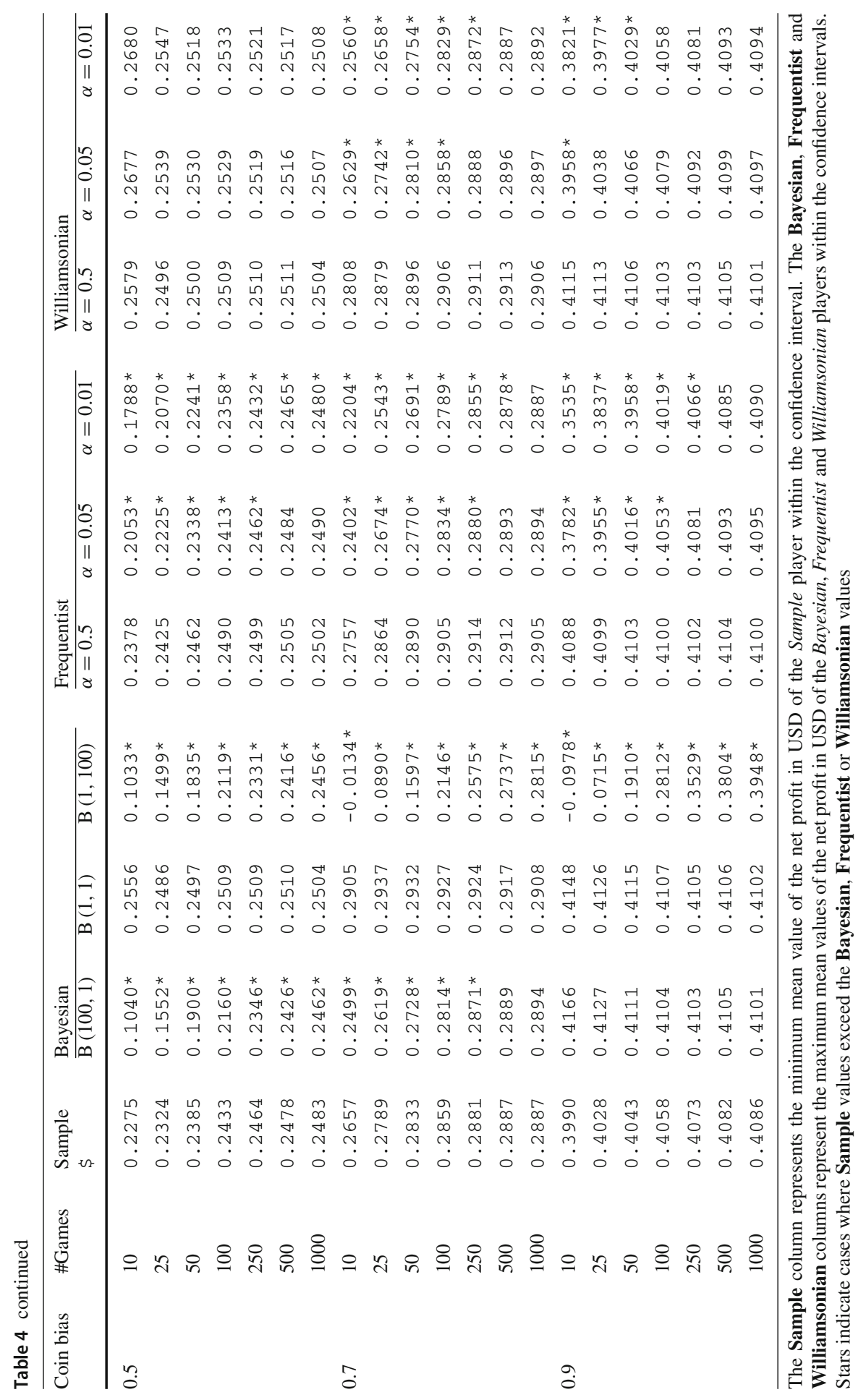




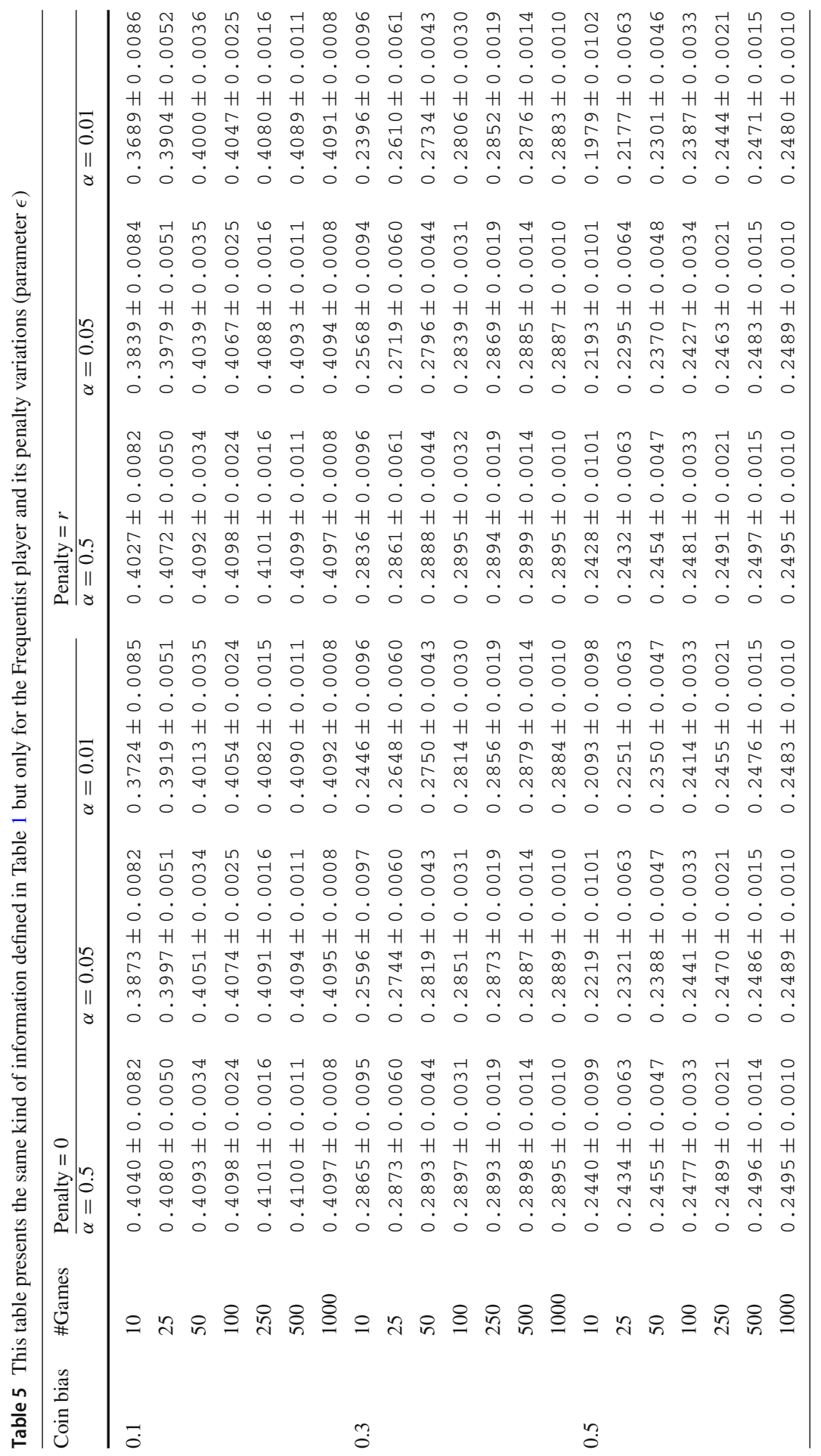




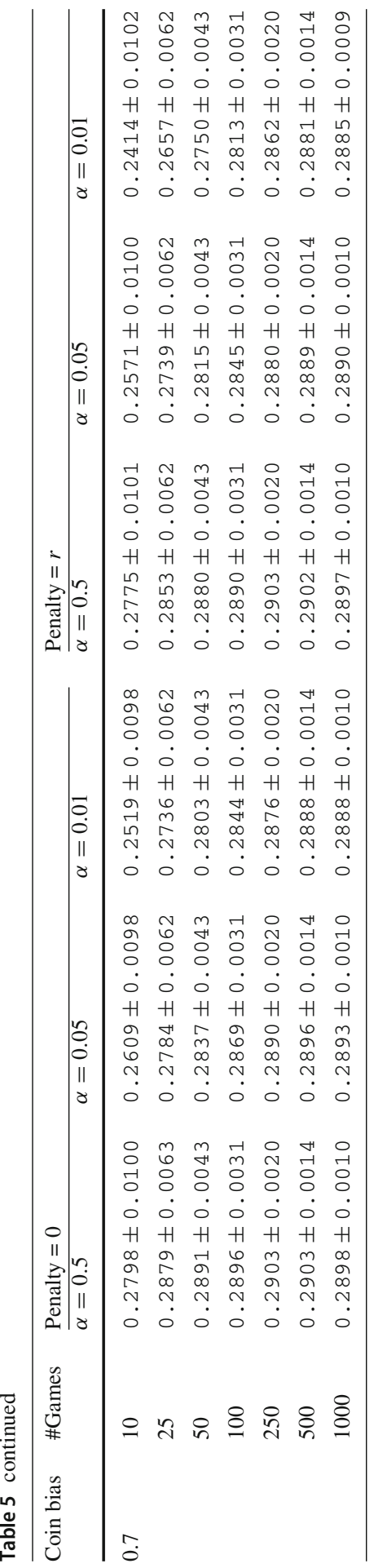




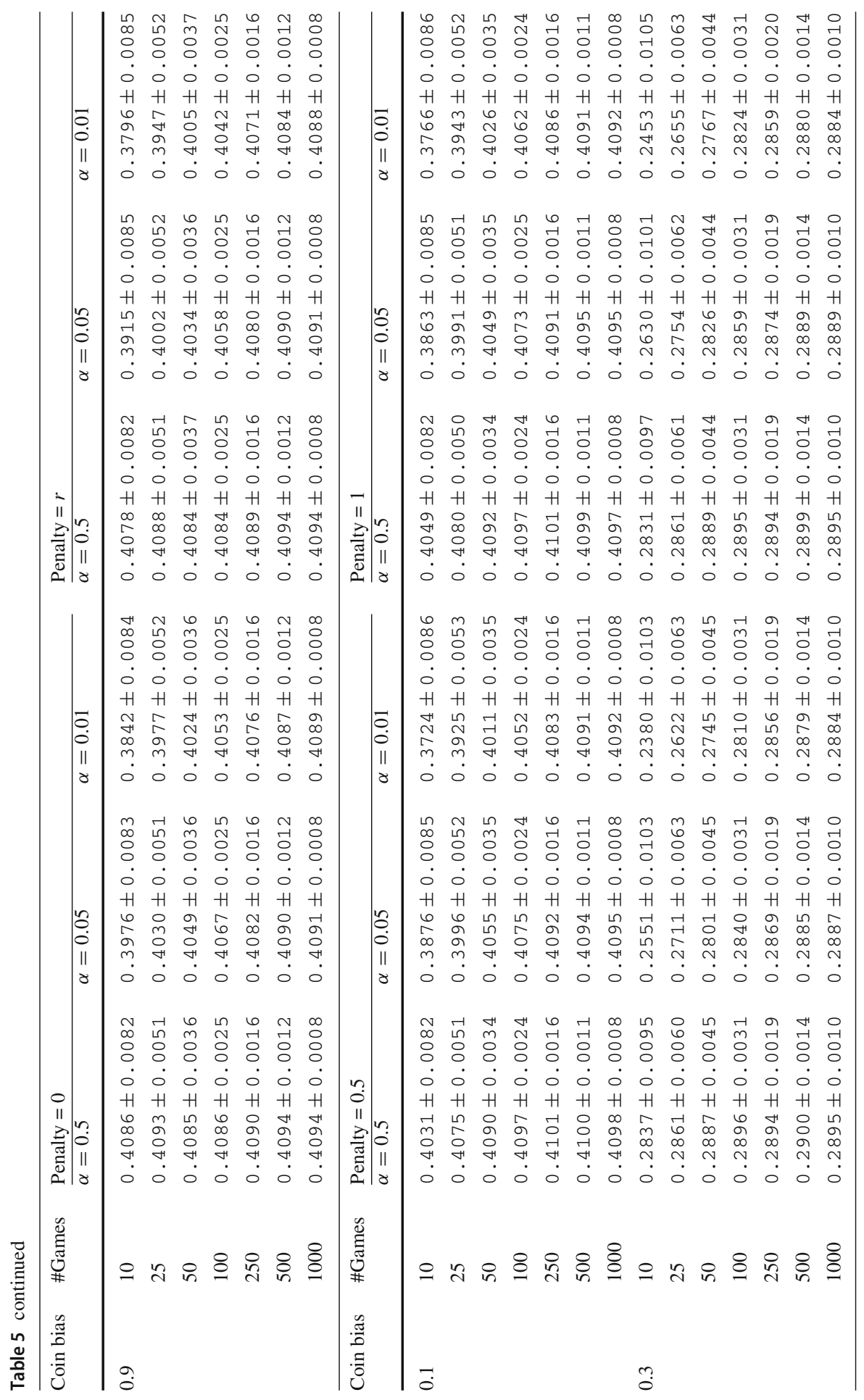




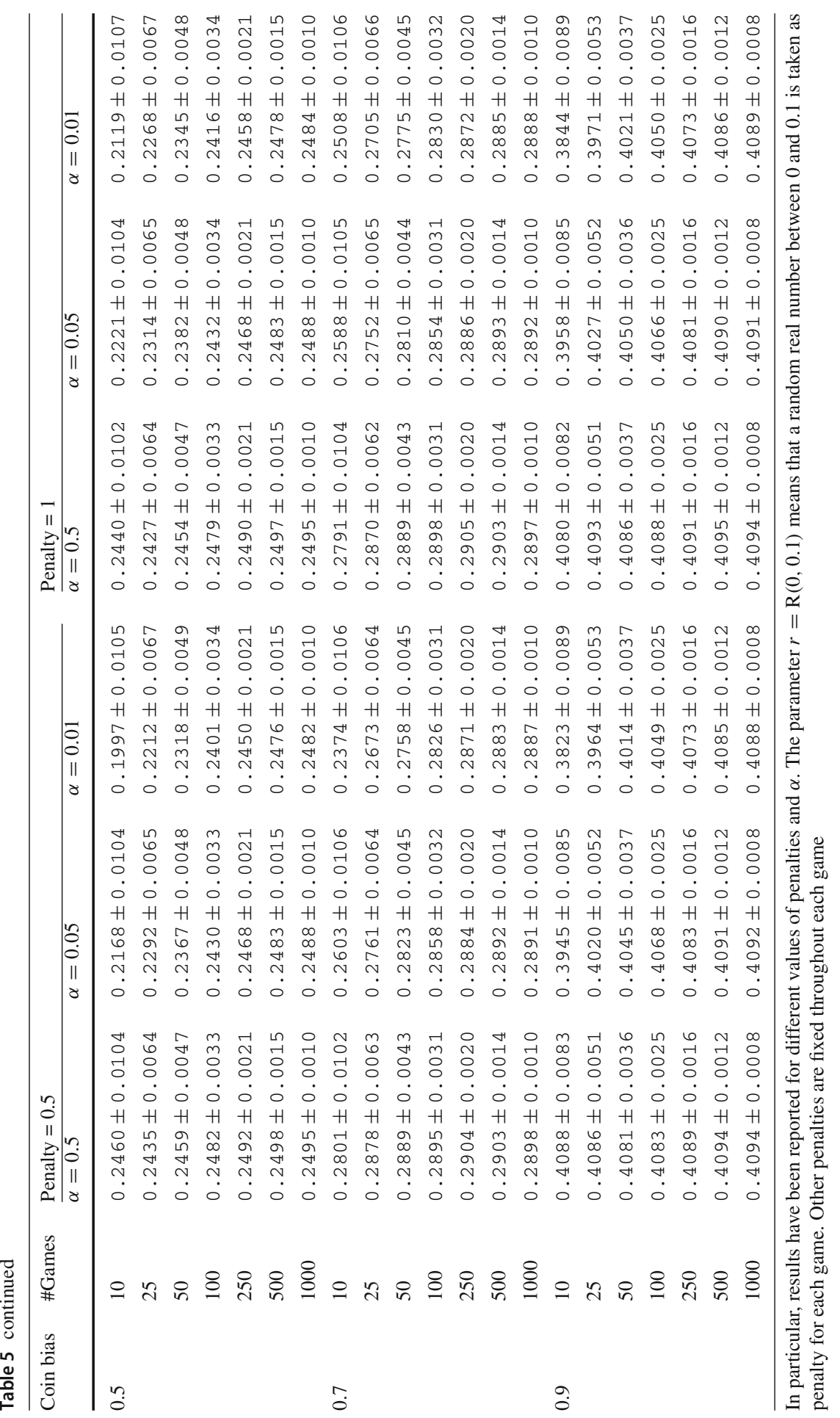




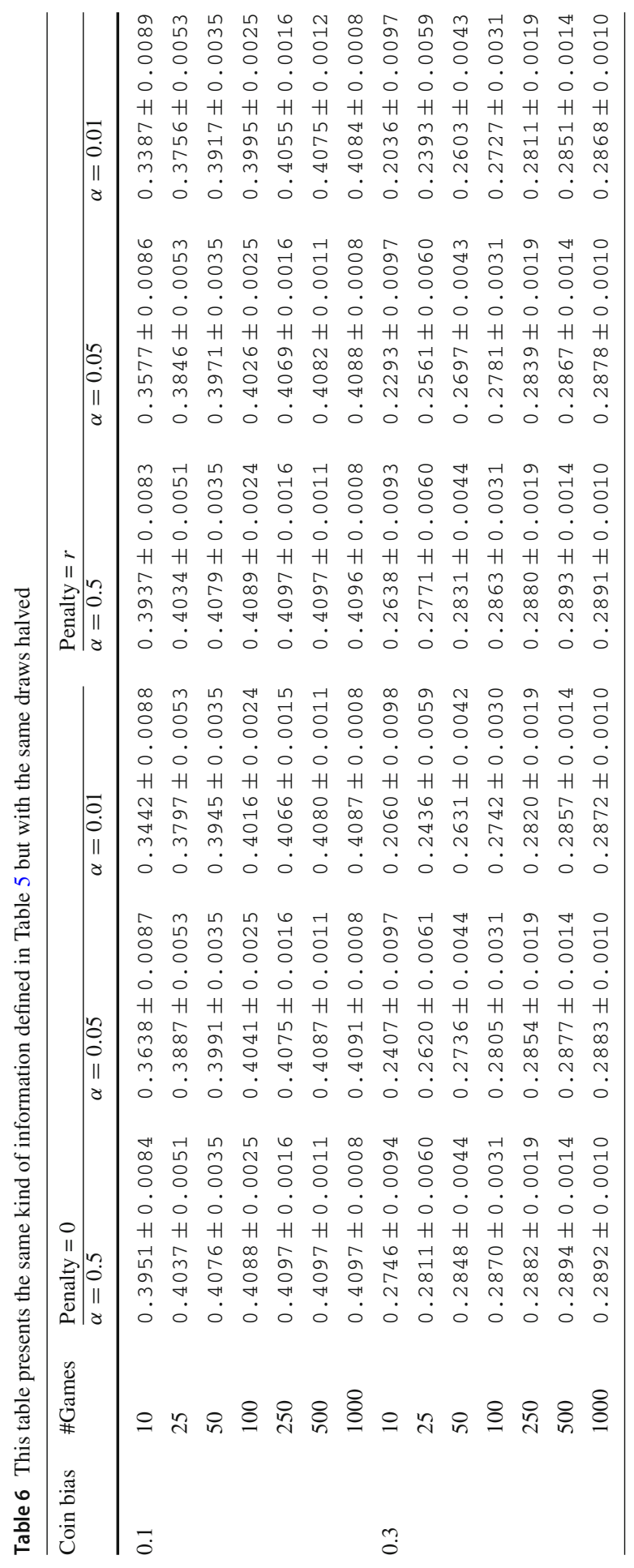




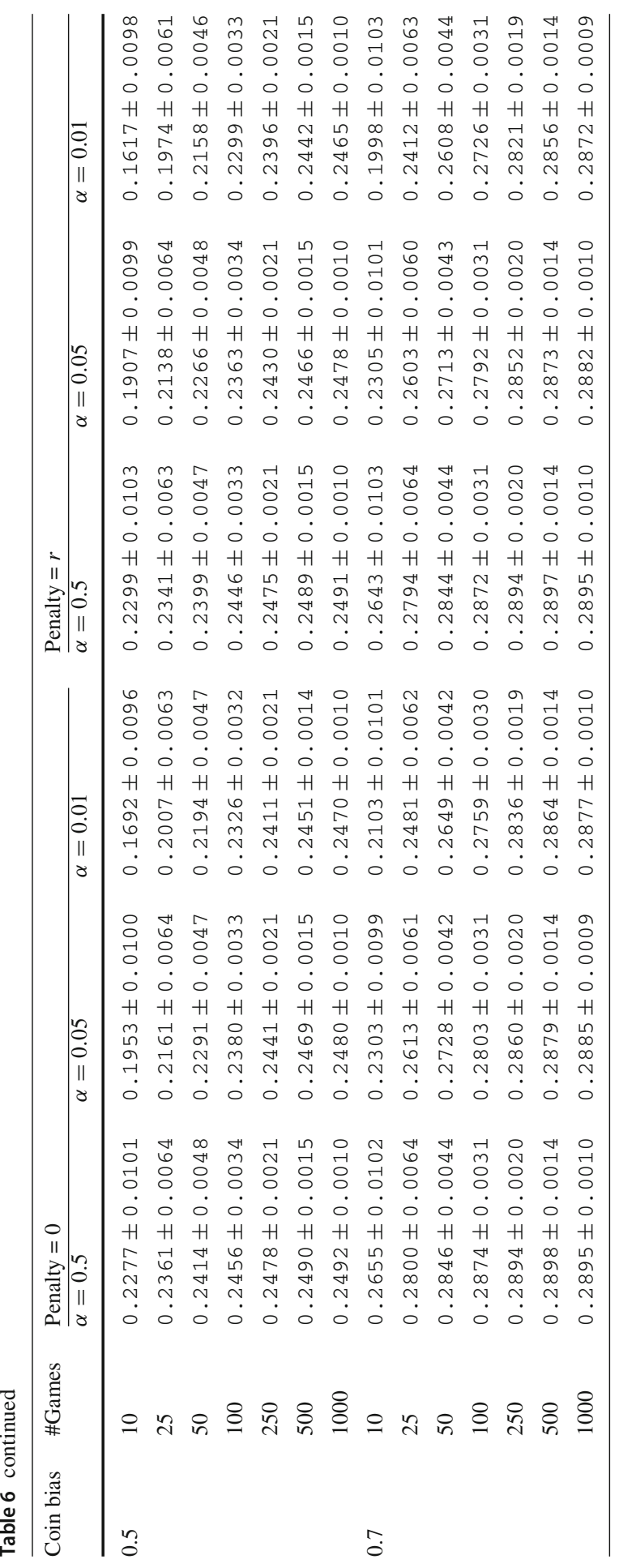




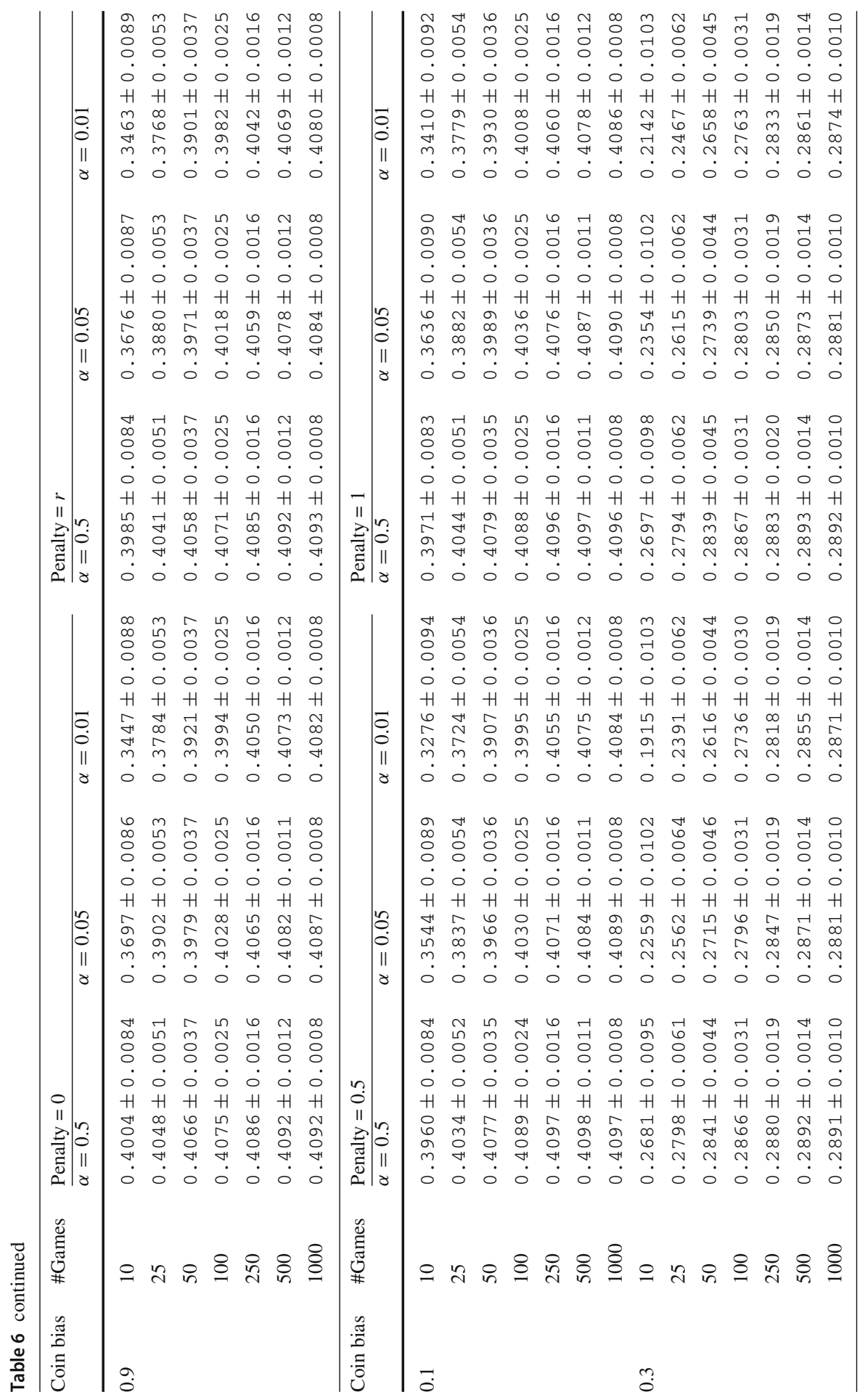




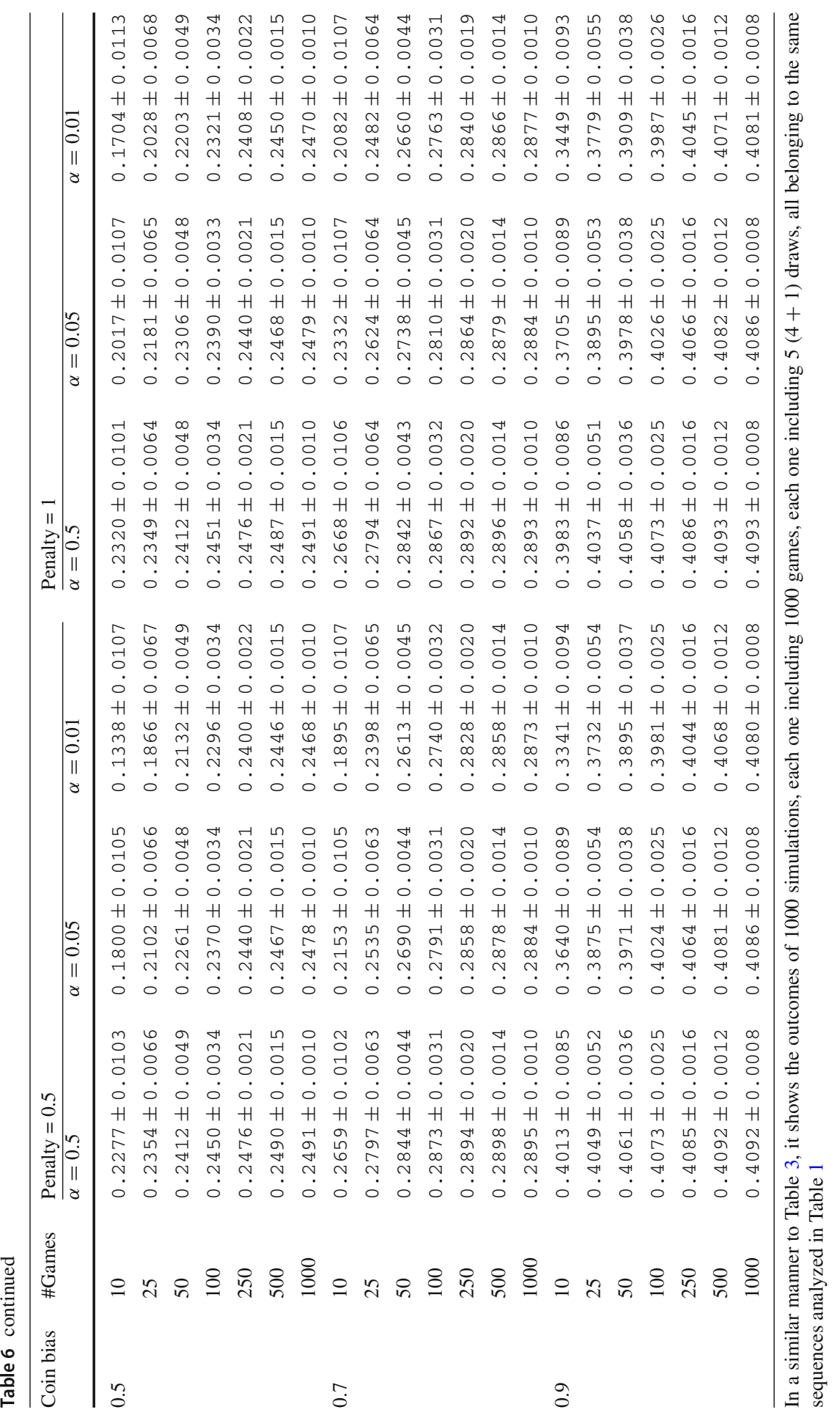




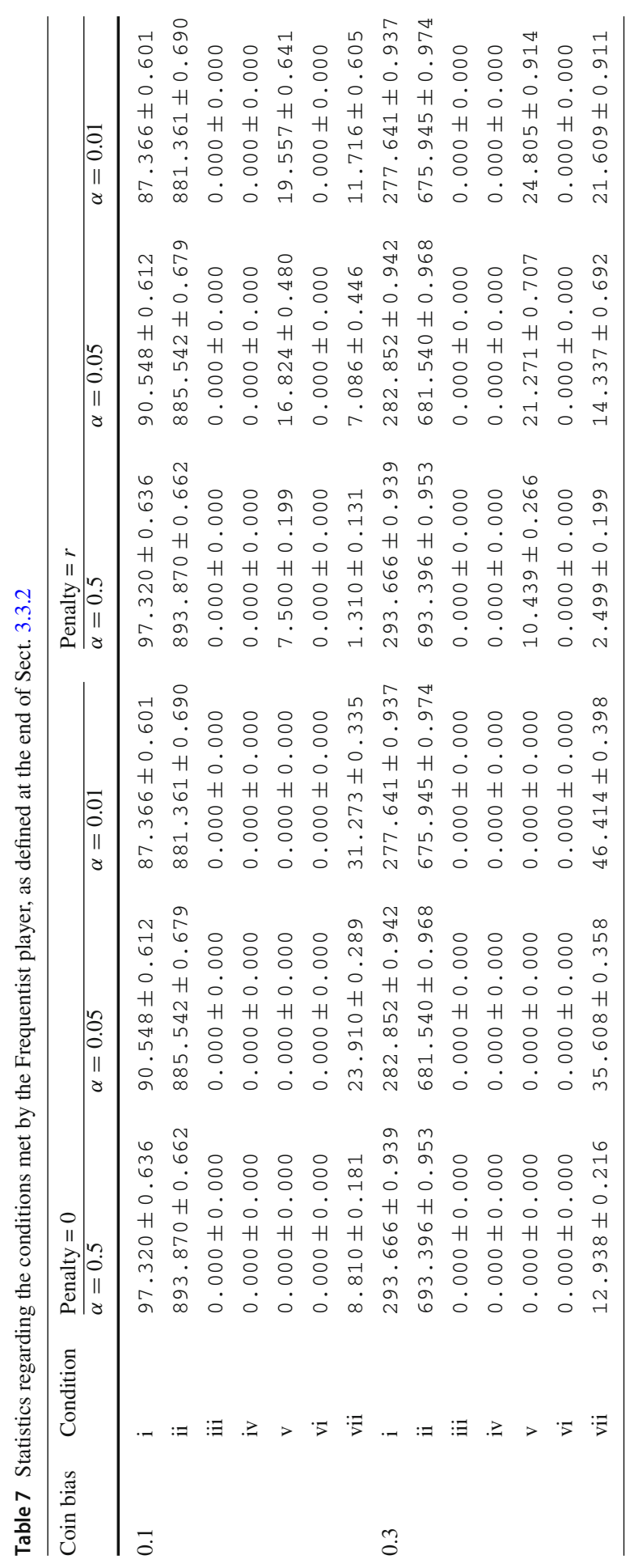




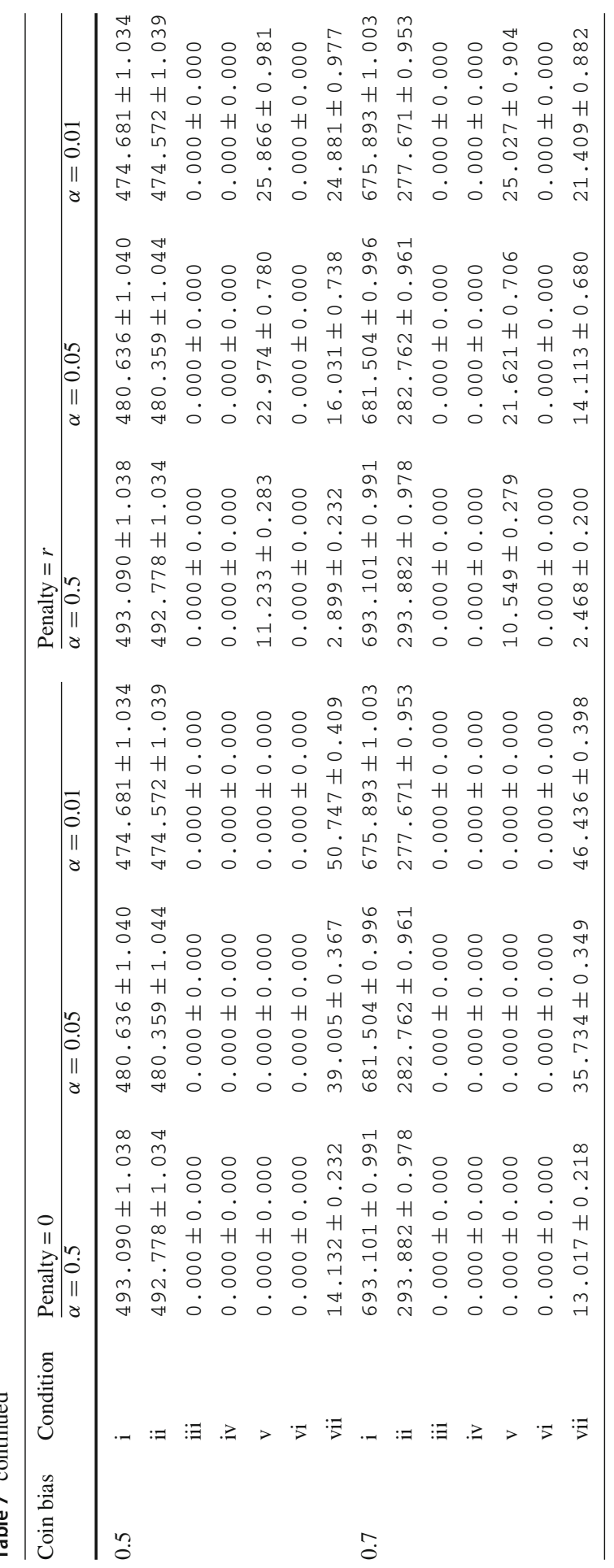




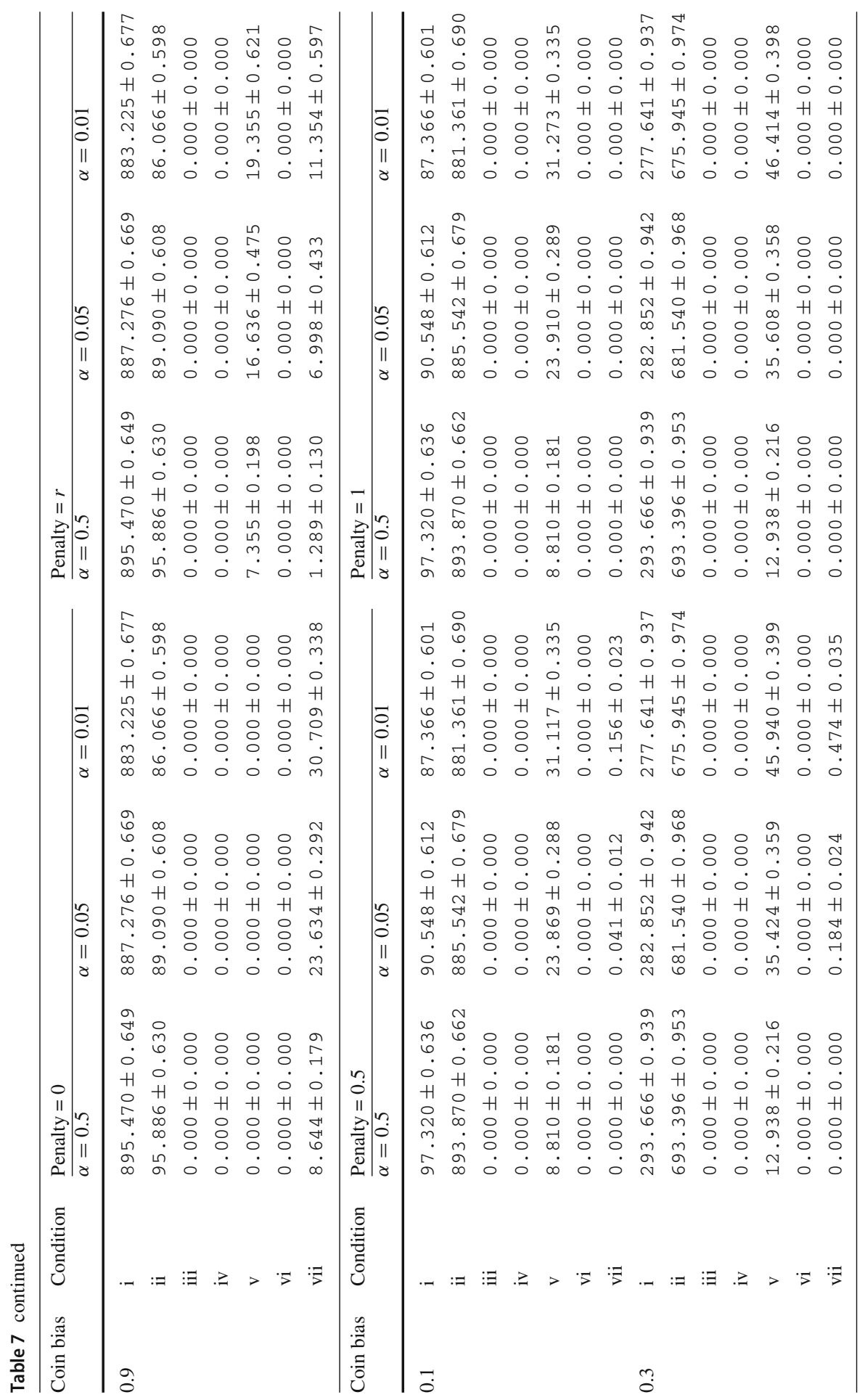




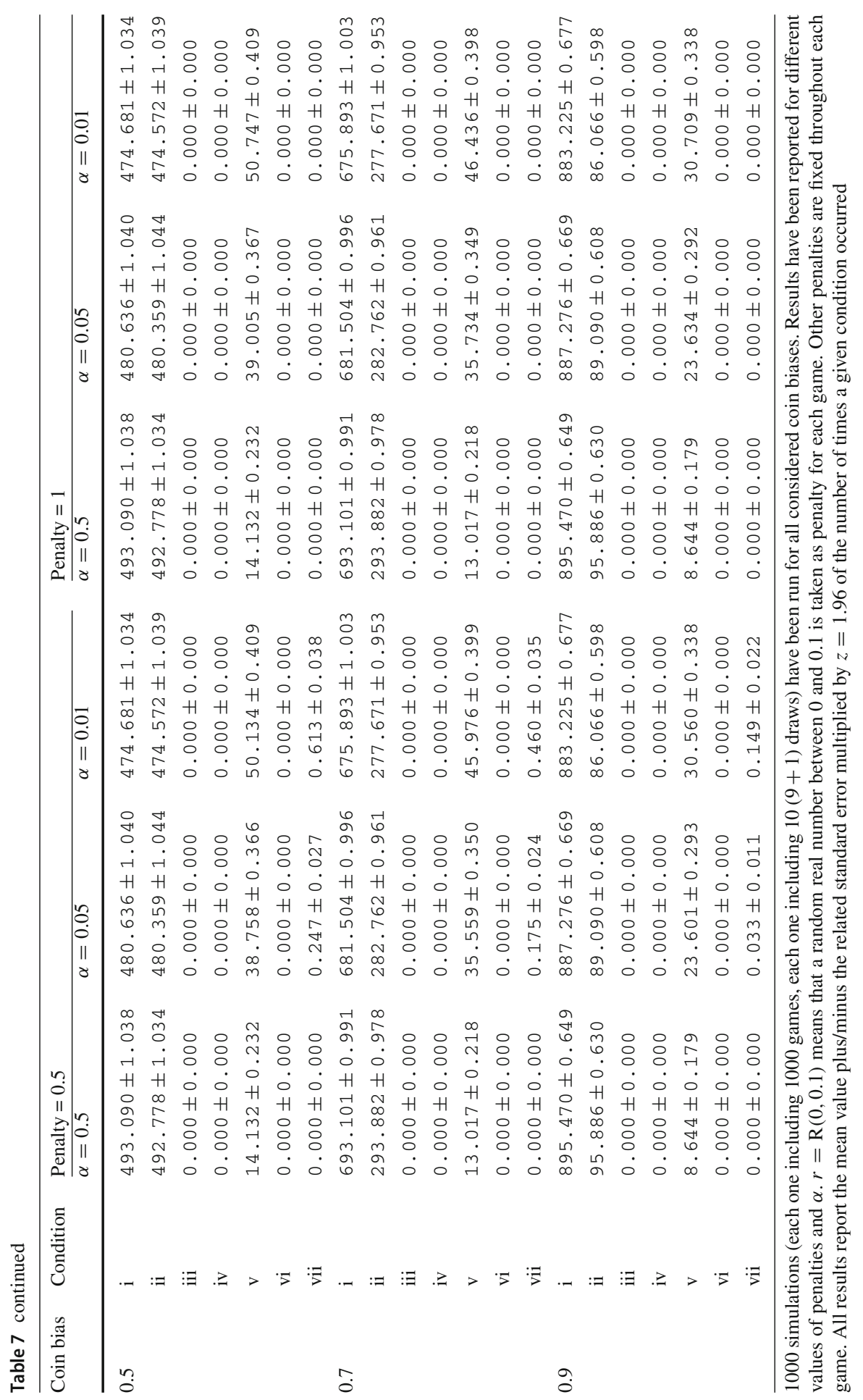


We made two sets of simulations. Each set consisted of 1000 simulations. In the first set of simulations, there were 1000 games. In each game, players observed 9 tosses and then bet on the 10th toss. In the initial game, players had no prior observations. They updated using their 9 observations and made a decision on how (and whether) to bet on the result of the 10th toss. After the 10th toss, players updated on the 10th toss. In subsequent games, retained knowledge of their past observations of tosses. Thus, for example, in a particular simulation, players had 500 observations after 50 games. In total, there were 1000 opportunities to bet per simulation, corresponding to the 1000 games.

In the second set of simulations, there were also 1000 games in each of the 1000 simulations. In these simulations, players had just 4 observations in each game, and they bet on the result of the 5th toss. The players' updating was otherwise identical to the first set of simulations. Therefore, after 50 games, players had 250 observations in a particular simulation. In total, there were 1000 opportunities to bet per simulation. We conducted this second set of simulations to investigate how players performed with very small samples. In each simulation, every player observed the same sequence of tosses.

For each of the 1000 games in a simulation we used 1000 randomly generated ticket prices. We used different ticket prices for each simulation. However, for a given simulation, every player faced the same ticket prices. We recorded the overall mean payoffs and standard deviations for each player in each simulation. We also recorded the overall mean payoffs and standard deviations at different points during the simulations, as we show in the tables below. Finally, we recorded how often Frequentist chose to bet and how often they chose to withhold from betting.

The sample sizes for players' observations ( 9 new observations per game for the first set of simulations, 4 for the second set) seemed to offer a reasonable balance between enabling us to look at short run performances and yet also giving players some data to use. No player will update their beliefs in a radical way in response to just several tosses. Therefore, with extremely small samples and just a few games, we would mainly be comparing the players' initial choice models-interesting in some respects, but not very informative about the differences in the players' statistical learning procedures. On the other hand, the players will almost always make identical decisions with very large samples, due to washing-out of priors and narrowing of confidence intervals. Our choice of sample sizes is intended to find a reasonable medium between comparisons with extremely large and extremely small samples.

In addition to varying the sample sizes, we also varied the coin bias across the values $0.1,0.3,0.5,0.7$, and 0.9 . For each simulation and each particular coin bias, we randomly generated a single history of coin tosses and simulated each player's behaviour with that history. Our choice to keep the histories fixed ensured that any differences in performance between players were not due to random variation in the coin toss histories that they faced.

We also made variations in players' exogenous parameters - the parameters in their belief and decision algorithms that are not set by the methodologies. For Bayes, we varied the values for the beta distribution parameters of $(a, b)$ to $(100,1),(1,1)$, and $(100,1)$. For Frequentist and Williamsonian, we varied the values for $\alpha$ to $0.01,0.05$, and 0.5. Finally, following the suggestion of Kyburg and Teng (1999), we varied the 


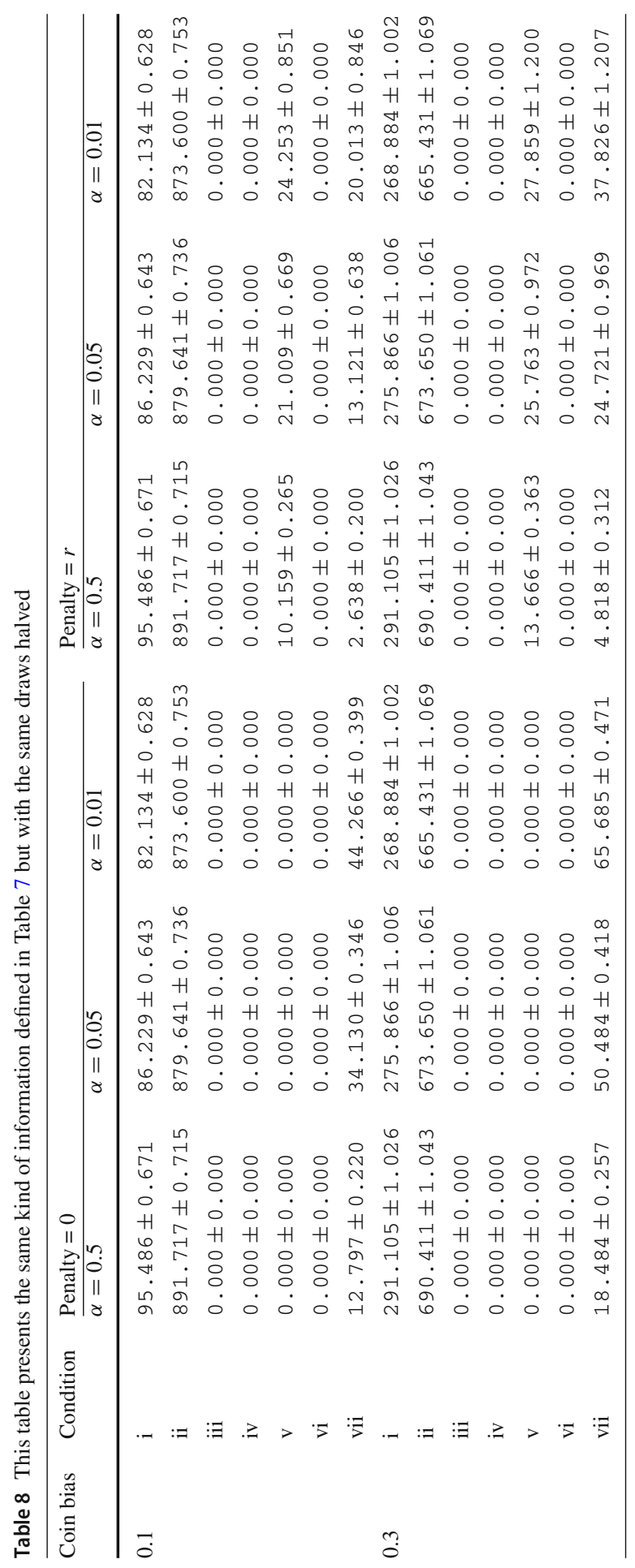




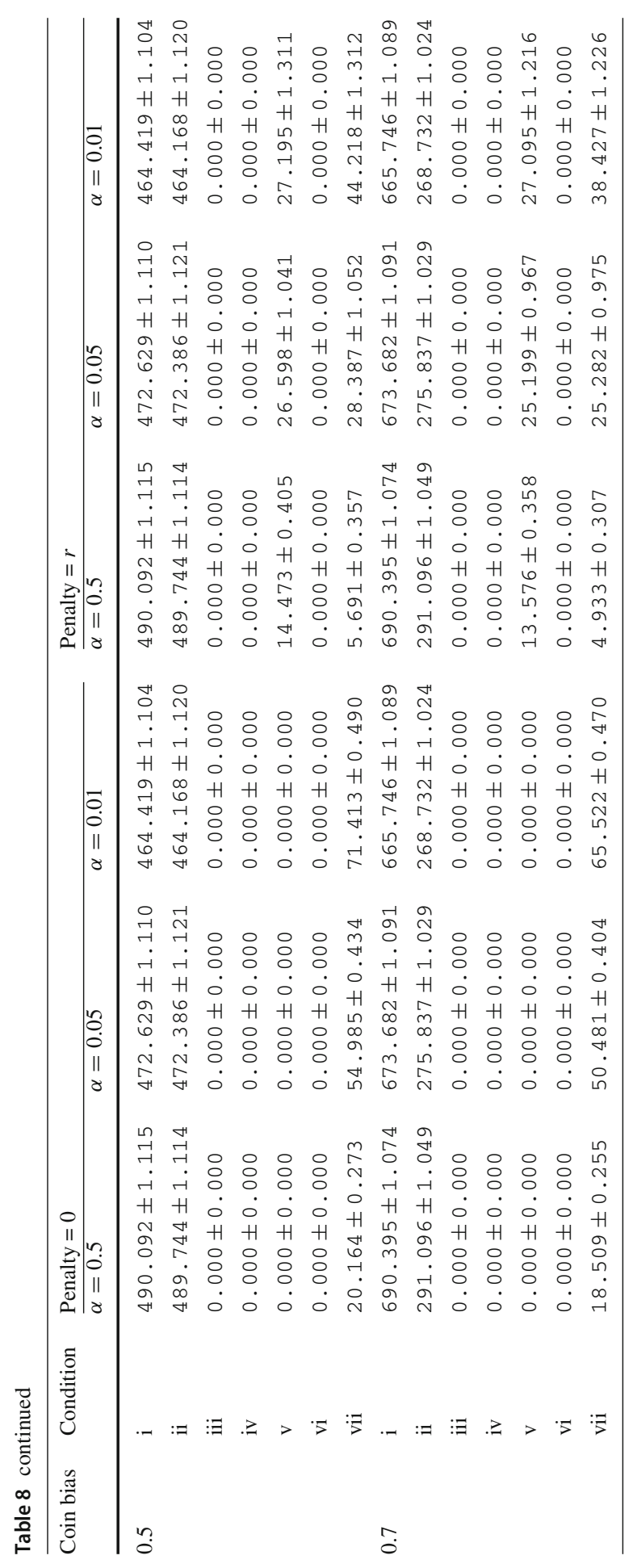




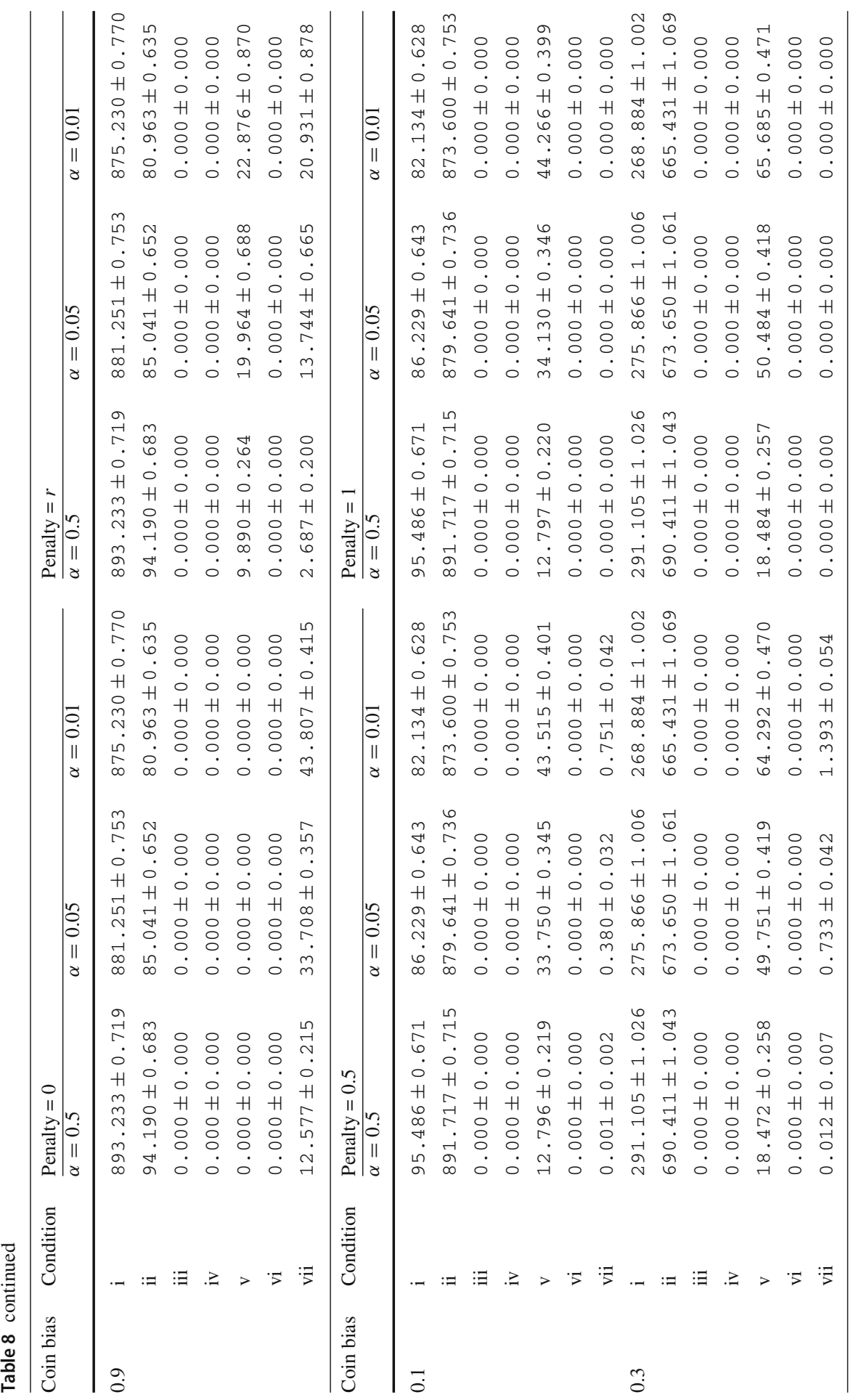




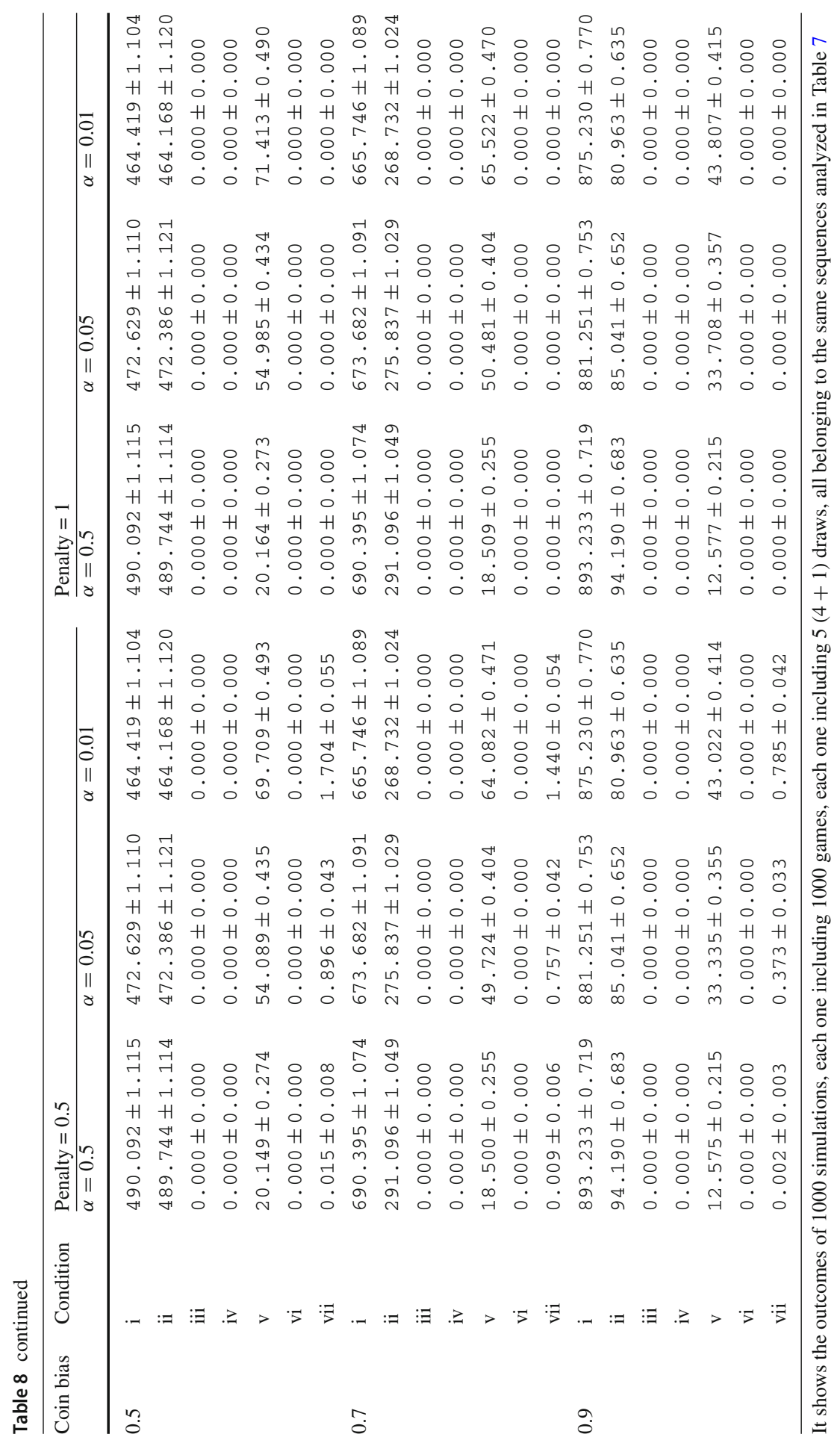


penalty parameter $\epsilon$ for Frequentist, with the intention of varying the willingness of Frequentist to place bets rather than hold. For Sample, there were no parameters to vary.

Each simulation's results were independent of every other simulation, and their basic stochastic parameters were the same for a given setting, e.g. a given coin bias. Consequently, our simulations might be regarded as independent and identically distributed draws from the overall population of possible simulations under these settings. In the next section, we use the standard error under that interpretation and a $95 \%$ confidence interval. However, we strongly stress our study is descriptive rather than inferential: we have provided some relevant descriptive statistics, but proper testing of hypotheses regarding our simulations is a project for further research. Like our simulation's code, our datasets are available upon request.

\section{Results and analysis}

We report our results in Tables 1, 2, 3, 4, 5, 6, 7 and 8. We begin with some comparative points, always using the standard errors reported in the tables. To evaluate the players in relation to the benchmark Sample player, we compared the best performance of a player in the confidence interval for that player's results with the worst performance of Sample in its confidence interval. Using this analysis, if a player fails to outperform Sample for a particular number of games (on average over the 1000 simulations) then we have starred the cell in Tables 2 and 4. In starless cells, the player outperforms Sample according to the interval analysis. This interval analysis acknowledges the random errors involved in such an assessment, while also indicating the descriptive basis for the evaluations that we make in this section.

When Bayes sets a $\mathrm{B}(1,1)$ prior $^{15}$ and Frequentist sets a high value of $\alpha$, we found that they both reliably either matched or exceeded the performance of the Sample player: see Tables 2 and 4 for $\mathrm{B}(1,1)$. In this respect, our results differ from those of Kyburg and Teng (1999). The very similar performance is unsurprising in a large number of games, where these players' performances were more or less identical. As sample sizes become large, then Bayes's posterior probabilities become very close the cumulative observed sample frequency, while Frequentist's confidence interval estimates are very narrow around that frequency. However, it is surprising with 10 games, where players had less than 100 observations to make their decisions in each game. Bayes with a $\mathrm{B}(1,1)$ prior and Frequentist with $\alpha=0.5$ are behaving differently, but neither performed detectably better across all the coin biases. The same was true for Williamsonian with $\alpha=0.5$. Overall, for each of the three statistical methodologies, there were player settings under which they passed the benchmark that we set.

We did find inferior performance relative to Sample when Bayes's beta prior was biased towards either heads, B(100,1), or tails, B(1,100). Indeed, in Tables 1 and 3, we can see that this player setting is the only one under which any player made a loss in the short-term. It is not surprising that, with a biased prior that differs from the coin bias, Bayes would perform badly. It is perhaps more surprising that these biases never

$\overline{15 \text { A beta prior with parameters } a}=1$ and $b=1$. 
yielded detectable special advantages relative to the flat $\mathrm{B}(1,1)$ prior. Even in the short run, there were no simulation settings where Bayes performed less well (in either the short run or the long run) than Sample using a B(1,1) prior but not with a biased prior for the 1000 simulations as a whole. Consequently, a biased prior created the risk of some very bad performances for Bayes, without identifiable benefits relative to flat priors. Furthermore, these costs of biased priors were persistent: even with thousands of observations over many games, the Bayes with a badly inappropriate prior was still performing badly. While a washing-out of priors would occur in the long run, it can take a long time in this sort of decision problem.

The absence of special benefits from a biased prior can be explained by the "flatness" of the $\mathrm{B}(1,1)$ Bayesian prior. Although this prior is an equivocal and thus would generally have some advantages for a 0.5 coin bias, it is not this equivocation that is the key to its success in our decision problem. Instead, the advantages come from the speed with which Bayes revises their beliefs using this prior. Suppose that the coin is biased towards heads, such that its frequency of heads is 0.9. Such a bias will tend to produce samples that are themselves usually biased towards heads, and the Bayesian will quickly update on this sample data if they have a $\mathrm{B}(1,1)$ prior, thus quickly eliminating any special advantages that the $\mathrm{B}(100,1)$ prior will have from being biased towards heads. Some other equivocal priors — e.g. a B $(100,100)$ prior-would not perform so well when observing the tosses of a biased coin.

In terms of common points, all players tended to do better when the coin bias was set further away from 0.5 . This result is unsurprising, because the chance of a very unrepresentative sample is greatest when the coin is fair. In contrast, if the coin bias is 0.1 or 0.9 , then the randomisation process in the Python code will generate very unrepresentative samples at a lower rate. Kyburg and Teng found the same result (Kyburg and Teng, 1999, pp. 362-363).

We now turn to particular players. As previously noted, Bayes did best with a $\mathrm{B}(1,1)$ beta prior. While this result might seem to be favourable to Objective Bayesian approaches, according to which such a prior would be mandatory, note that Subjective Bayesians regard flat priors as permissible, provided that they are coherent with the total credence distribution. They just deny that they are rationally required. Furthermore, as a matter of contingent fact, most real-world Subjective Bayesian statisticians would choose such a prior if faced with the decision we describe. Since Bayesian reasoning with such a "flat" prior is similar to maximum likelihood estimation, it is also unsurprising that Bayes with a $\mathrm{B}(1,1)$ beta prior would at least match the performance of Sample.

We now consider the Williamsonian. They differ from the players studied in Kyburg and Teng (1999). Although Williamson's theory of statistics is not as prominent as the Bayesian or frequentist methodologies, it provided us with the basis for an intriguing "hybrid" player. ${ }^{16}$ Notably, Williamsonian matched the performance of Bayes and Frequentist. In some cases, with low values of $\alpha$, the Williamsonian failed to match the performance of Sample in the very short run. However, this varied with different coin bias settings, so it was not a consistent pattern. Much more investigation is needed

\footnotetext{
16 We stress that it was inspired by Williamson's views; we do not presume that Williamson would agree with every detail of it, anymore than all Bayesians or all frequentists will approve of Bayes and Frequentist respectively.
} 
to infer anything definite about the best values of $\alpha$ for Williamsonian's performance. The problem might be that low values of $\alpha$ increase the importance of the Equivocation norm as their confidence intervals will detect coin biases more slowly. If the coin bias is 0.5 , then this behaviour is unproblematic: see Tables 2 and 4. However, when the coin is biased, the Equivocation norm can repeatedly drag Williamsonian's credences towards 0.5. Our results are consistent with Williamson's principal justification for the Equivocation norm: its advantages in minimising loss for the worst-case scenarios (Williamson, 2007). For combinatorial reasons, 0.5 is the least favourable stochastic condition for binomial sampling, because it maximises the number of unrepresentative samples.

At least in our simulations, Williamsonian was able to "have their cake and eat it" by using a (maximally) low value of $\alpha=0.5$. They were still able to match or exceed the performance of Sample when the coin bias was 0.5, in both the short and long run. Yet the greater receptiveness to sample data when $\alpha=0.5$ meant that they were also able to pass our benchmark with other coin biases. Nonetheless, one cannot extrapolate to all decision problems and say that a low value of $\alpha$ will always be better for Williamsonian. In some decision problems, perhaps with rare extreme risks, being firmly equivocal could be beneficial.

Regarding biased priors, Williamsonian excludes these via the Equivocation norm, and thus they do not face the problem of having such a prior in this decision problem. Consequently, even the worst performances of Williamsonian were not as bad as the worst performances of Bayes with a prior that was biased in the wrong direction. For instance, see Table 4 , for coin bias $=0.1$ or 0.9 , and runs of 10 games, where Williamsonian with $\alpha=0.01$ does far better than Bayes with $\mathrm{B}(100,1)$.

Finally, we consider Frequentist. For $\epsilon$, we firstly found that this parameter achieved its intended function of increasing Frequentist's propensity to bet, as we report in Tables 7 and 8. On the other hand, $\epsilon$ does not seem to reliably affect Frequentist's performance, as we see in Table 6. As for $\alpha$, we found the same result as Kyburg and Teng (1999). In the very short run, there were some suggestive but indefinite signs that very low values of $\alpha$-particularly $\alpha=0.01$ - could lead to a poor performance relative to Sample, but more research is needed; the differences were not as clear as with Bayes using a biased prior. For $\alpha=0.05$ and $\alpha=0.5$, there were at least some occasions where Frequentist matched Sample. When $\alpha=0.5$, this above-benchmark performance was consistent.

Why might high values of $\alpha$ hurt the performance of Frequentist? This question was not a focus of our study and thus it would benefit from more comprehensive analysis. Nonetheless, one notable point from Tables 7 and 8 is that a lower value of $\alpha$ made an identifiable difference to the rate of using randomisation. Recall that, in Frequentist's mixed strategy decision rule, they randomise between buying a ticket, buying a reversed ticket, or withholding from betting in situation (vii), and between buying a ticket or a buying a reversed ticket in situation (v), as we detail in Sect. 3.3.2. ${ }^{17}$ In situation (v), neither betting heads nor betting on tails interval dominates the other given Frequentist's estimated confidence interval. Thus, they randomise between these two

\footnotetext{
17 Due to the incentive structure of the game, there was no occurrence of situations (iii), (iv) and (vi), which correspond to cases where withholding interval dominates at least one other action. This clearly appears also in Tables 7 and 8.
} 
bets using an additional fair coin. In situation (vii), none of their three possible actions is interval dominated, and therefore Frequentist randomises between all three. Both these randomisation procedures mean that Frequentist is acting equivocally between heads and tails. Consequently, if $\alpha$ is low, then they are not making much use of sample data indicating bias, but if $\alpha$ is high, then Frequentist quickly detects bias and exceeds the minimum performance of Sample even in the very short run, as in Tables 2 and 4. It does not follow that a high value of $\alpha$ is better for decision problems in general. Instead, our results suggest high values are better for this kind of decision problem, because it makes prompter use of the sample information, and hence helps avoid acting equivocally when the coin is biased.

\section{Discussion}

Although our simulations did not detect any reason to use one statistical methodology rather than another, it does not follow that the choice is underdetermined. Firstly, Bayesians might note that, unlike Bayesian decision theory, Frequentist's decision algorithm has no axiomatic derivation; Kyburg acknowledges that it is produced by unsystematic intuitive considerations (Kyburg, 2003, p. 148). We were able to use these intuitive considerations to infer how Frequentist should extend the algorithm from Kyburg and Teng (1999) to cases where there was a non-zero penalty for holding, but it would be an extreme exaggeration to call it a decision "theory". More generally, there is no agreement on how to make decisions with interval-valued beliefs. ${ }^{18}$ Secondly, frequentists might argue that there are broader aspects like long run decisions, social decisions, or epistemological points that we do not address in our simulations, which only involve individual decision-making in the short run. Thirdly, Williamsonians also think that their approach has long run virtues that favour their view for decisionmaking (Williamson, 2007). Our results suggest that either (1) we need a different sort of challenge for comparing their short run performance or (2) the choice between them-even when we focus on the relatively narrow issue of making good decisionsmust be approached via questions of systematic coherence, the social consequences of their implementation in science, asymptotic strengths or weaknesses, results of debates in formal learning theory (Schulte, 2018) and other angles.

Our results have some good news for all three methodologies. For Bayesians, our results are better than those of Kyburg and Teng (1999). Unlike their study, we did not find that the Bayesian player will generally be outperformed by the frequentist player. For frequentists, they can take comfort from the fact that Frequentist can match Bayes in performance, even when the Bayesian's prior is set to the true relative frequency, and even though guiding us to good decisions is often regarded as a strength of Bayesianism. Finally, for Williamsonians, we have shown how they can match the performance of the more well-known Bayesian and frequentist methodologies. This strong performance by Williamsonian might encourage a wider audience of philoso-

\footnotetext{
18 For some discussions, see Kyburg (1990), Seidenfeld (2004), Troffaes (2007), Haenni et al. (2011), Bradley (2017).
} 
phers, statisticians, and other interested groups to inquire further into the relatively marginal Williamsonian position.

The similarity in performance is particularly intriguing because it is not always caused by players making the same decisions. It is true that, in the long run, players have almost identical performances because they have more or less the same beliefs, and they are making the same decisions. That occurs because, with large samples, the initial priors lose their significance; the confidence intervals narrow; and the players concur on the best decisions for the greater proportion of a given coin toss history and sequence of ticket prices. However, with smaller samples, the players have different beliefs and make different decisions, which is why their results vary among particular coin toss histories. The lack of a systematic difference is thus occurring in spite of the players' contrasting decisions. Therefore, in the short run, the players have genuinely different statistical methodologies, but in our problem, their methodologies do not guide them to relatively better decisions.

The problem of priors is also a heated topic within the Statistics Wars. Overall, Bayes did badly with an extreme prior, and their performance was no better (relative to Sample) than the Bayes setting $\mathrm{B}(1,1)$, even when their biased prior was in the right direction relative to the coin bias. However, one point that our simulations illustrate is that the effects of "washing-out" of the priors can take a long time to manifest. In the long run, we would expect to see Bayes catch up even with a very "bad" prior. Nonetheless, in the real world, relative performance in the short run can make an important difference for aims like attracting investment in business, attracting funding in science, or winning an arms race. ${ }^{19}$

Moreover, the decision problem and priors we used is arguably favourable to Bayes's catching up in such cases. Bayes views the coin tosses as an exchangeable. This means that the tosses are independently and identically distributed according to some distributional form, which implies that the order of the tosses does not matter. More fundamentally, their prior was always sensitive to evidence. Subjective Bayesianism as such does not require such priors. Most obviously, extreme priors of 0 or 1 cannot be changed by conditionalization. However, the further possibility of non-extreme priors that share this dogmatism have been known since the early days of Bayesian epistemology (Carnap, 1945a, pp. 80-81). The limits of what can be learned by conditionalization has been a topic of research in recent formal epistemology (Rédei and Gyenis, 2019). For instance, if a Bayesian player was estimating the general relative frequencies in a massively large number of tosses, then clearly there are prior distributions that will be totally insensitive to new evidence. Thus, we recognise that it would be possible to create tougher learning challenges for the Bayesian-just as it may be possible to construct choice problems where the frequentist decision theory we consider entails inconsistent or systematically exploitable choices. On the other hand, it is possible that Bayesians might do better in problems involving more background knowledge than the sparse information that they (and the other players) possess in this article's simulations.

\footnotetext{
19 In general, relative short run performance is very significant for problems that are analogous to being at the front of a stampede of animals, where it is your relative speed that determines whether you are trampled.
} 
The use of the penalty $\epsilon$ enabled us to remove an asymmetry between the Bayesian and frequentist players in Kyburg and Teng (1999). In their simulations, the frequentist player would refuse to bet until they either had relatively large samples or they were faced with very attractive (given their beliefs) ticket prices. In contrast, their Bayesian player would always bet, even with very small samples. It was possible that this asymmetry produced Kyburg and Teng's favourable results for the frequentist. Perhaps surprisingly, in our simulations, forcing Frequentist to bet using $\epsilon$ made no systematic difference to their performance relative to Sample. However, there is still a possibly important asymmetry when $\epsilon$ is high. Frequentist, when forced to bet, will randomise unless they have either relatively large samples or very attractive ticket prices. In contrast, Bayes almost never randomises, because they will only randomise if two bets have the same expected payoff. One might think that this reflects a methodological difference, in which case this asymmetry is not a problem for our comparisons. However, it indicates the dangers of generalising from our results to other decision problems, such as those in which there is a yet another penalty, this time directed at randomisation. Additionally, one reason for thinking that the asymmetry is not due to anything specific to the methodologies is that other types of prior for Bayes could reduce this asymmetry, even within our setup. For instance, Bayes could have an equivocal prior that was "sticky" in the sense of not changing (or not changing much) until they had relatively large samples. While a beta distribution was a reasonable way to model Bayes, there is nothing in Subjective Bayesianism that mandates this family of priors. Frequentist could also be modified to randomise less, by giving them a rule like maximin, which would involve less randomisation than the interval-dominance that we used in this study. We leave such alternative simulations for future research, but their possibility should warn against generalising from our simulations to other decision problems or different player settings.

Another contrast with Kyburg and Teng (1999) is that we have extended the simulations in some new ways. In addition to those already mentioned (such as the addition of Williamsonian) we have explored the players' performances in relatively large numbers of games-while Kyburg and Teng (1999, p. 362) stopped after 50 games, we have compared players' performances over 100, 250, 500, and 1000 games. We thus investigated both the relatively short and the medium run. Even though our primary interest was short run performances, the latter provides a useful check of both our model (if we expect convergence of performance in the long run) and whether clear differences emerged in the long run.

The principal divergence of our results and those of Kyburg and Teng (1999) is that we do not find, for any setting, that Frequentist detectably outperforms Bayes. We can explain the results in Kyburg and Teng (1999) in terms of randomness. They performed only 100 simulations, so the modest differences between players in their results can easily be explained by random error. By performing many simulations, we have greatly reduced the effects of randomness on our results. Their study retains some interest as a possibility result: our results do not contradict the possibility that Frequentist will sometimes perform better than Bayes in a relatively small number of simulations. What we have found is that such an outcome does not systematically occur for the best settings of these players out of the settings that we investigated. 
Without an extensive theoretical analysis, we can only speculate briefly on why (with the appropriate settings) the players had similar results. Relative to Sample, the best performing players were Bayes with a $\mathrm{B}(1,1)$ prior, Frequentist with $\alpha=0.5$, and Williamsonian with $\alpha=0.5$. Their performances were very similar under every coin bias and with either games with 5 tosses or with 10 tosses. How can this similarity be explained?

For these three player settings, a common trait is their use of sophisticated inductive inference from their evidence. Like Sample, they are all willing to revise their beliefs quickly given the background knowledge and evidence that we gave them in our study. In the case of Frequentist and Williamsonian, this readiness was due to the low value of $\alpha$, which respectively reduced the importance of randomisation and Equivocation. For Bayes, this readiness was due to a flat prior. Yet, unlike Sample, their early decisions were moderated by some factor that encouraged equivocal (and thus asymptotically cautious) reasoning in the early part of the game, thus downplaying the importance of early uniform samples. For Bayes and Williamsonian, this equivocation is achieved via the determination of their degrees of belief, using the beta prior and the Equivocation norm respectively. For Frequentist, it is achieved in their decision-making procedure: recall that their mixed strategy requires randomisation using a fair coin when no action interval-dominates any other; this underdetermination occurs most often early in the simulations, when sample sizes are small and confidence intervals can be wide even with $\alpha=0.5$.

Hence, for philosophers, our results indicate the power of sophisticated statistical induction, in the sense of (1) revising one's initial beliefs about the world to be closer to observed frequencies, but also (2) not extrapolating too strongly from small samples. Sophisticated induction can be achieved by multiple methods, including as conditionalization and confidence interval estimation. It then only needs an adequate decision procedure, and we found that both maximising expected payoffs (the approach of Bayes and Williamsonian) and the interval dominance rule of Frequentist were adequate for the problem we studied, provided that the player's other settings were also adequate.

\section{Conclusion}

Our simulation study had the result of a Caucus Race (Carroll, 1920, p. 34), in which "everybody has won, and all must have prizes." This result obtained even in the very short run, before convergence theorems could manifest. In contrast to Kyburg and Teng (1999), we did not find that the frequentist player will generally outperform the other players. However, contrary to what some philosophers might expect, our frequentist player did no worse than the Bayesian. We also found that this result obtained after we introduced new features to the simulations, such as a penalty for not betting that encouraged Frequentist to place more bets. We also introduced a hitherto unstudied player, based on Williamson's version of Objective Bayesianism, and we found that they performed just as well as the more well-known methodologies.

There are many limitations of our simulations that should be noted in order to avoid overgeneralisations. We simulated just one type of decision problem. The data and the 
problem itself were both very simple. The players could also be modified in ways that would be consistent with the statistical methodology that inspired them. We also only considered one algorithm for Frequentist and this algorithm lacked a systematic derivation from a generally accepted non-Bayesian theory of decision, because the latter does not exist. Another issue is that, like Kyburg and Teng (1999), our players differ in both their inference rules and their decision rules. ${ }^{20}$ We analysed our results informally; we welcome the use of either our results or code for more systematic analyses.

The type of simulations we conducted are largely unstudied and there are many novel dimensions for further exploration. It would be interesting to investigate more alternative players. For example, there are many, many types of Bayesians: how would Imprecise Bayesians, whose beliefs are characterised by sets of probability functions, perform in comparison to Bayes? How would Frequentist perform with other decision algorithms? The game set-up could also be modified in various ways. Currently, it features a relatively simple problem space and simple data, but would the players still match each other's performance when faced with more complex challenges? What if we make the game interactive? These directions indicate the fertility of this field of research.

The strong performance of all four players in our study should remind philosophers and those working in applications of statistics to respect the capacity of Bayesianism, frequentism, and Williamsonianism to guide us towards good decisions. Our little skirmish does not win the Statistics Wars for any side, but it indicates that all sides have firepower that is worth taking seriously.

Acknowledgements We thank Daniele Tortoli (University of Modena and Reggio Emilia, Italy) for his very valuable support in accelerating computations in our research. We are grateful to two anonymous referees for their helpful comments. We also thank the Erasmus Institute for Philosophy and Economics for their advice on the development of this article. Likewise, we thank Durham University for providing open access for this article.

Open Access This article is licensed under a Creative Commons Attribution 4.0 International License, which permits use, sharing, adaptation, distribution and reproduction in any medium or format, as long as you give appropriate credit to the original author(s) and the source, provide a link to the Creative Commons licence, and indicate if changes were made. The images or other third party material in this article are included in the article's Creative Commons licence, unless indicated otherwise in a credit line to the material. If material is not included in the article's Creative Commons licence and your intended use is not permitted by statutory regulation or exceeds the permitted use, you will need to obtain permission directly from the copyright holder. To view a copy of this licence, visit http://creativecommons.org/licenses/by/4.0/.

\section{References}

Bacchus, F., Kyburg, H. E., \& Thalos, M. (1990). Against conditionalization. Synthese, 85(3), 475-506. https://doi.org/10.1007/bf00484837

Benenson, F. C. (1984). Probability, objectivity and evidence. Routledge and Kegan Paul.

Bradley, R. (2017). Decision theory with a human face. Cambridge University Press. https://doi.org/10. $1017 / 9780511760105$

20 We are grateful to an anonymous referee, who points out that the aforementioned "imprecise" Bayesian approach offers a neater comparison with Frequentist in this respect, which provides a further reason to examine such a player in future research. 
Carnap, R. (1945a). On inductive logic. Philosophy of Science, 12(2), 72-97. https://doi.org/10.1086/ 286851

Carnap, R. (1945b). The two concepts of probability: The problem of probability. Philosophy and Phenomenological Research, 5(4), 513. https://doi.org/10.2307/2102817

Carnap, R. (1952). The continuum of inductive methods. The University of Chicago Press.

Carroll, L. (1920). Alice's adventures in wonderland. The Macmillan Company.

Celeux, G., Anbari, M. E., Marin, J. M., \& Robert, C. P. (2012). Regularization in regression: Comparing Bayesian and frequentist methods in a poorly informative situation. Bayesian Analysis, 7(2), 477-502. https://doi.org/10.1214/12-ba716

De Finetti, B. (1980). Foresight: Its logical laws, its subjective sources. In H. E. Kyburg Jr. \& H. Smokler (Eds.), Studies in subjective probability. Krieger Publishing Company.

Feigl, H. (1954). Scientific method without metaphysical presuppositions. Philosophical Studies, 5(2), 17-29. https://doi.org/10.1007/bf02223254

Fidler, F., \& Wilcox, J. (2018). Reproducibility of scientific results. In E. N. Zalta (Ed.), The Stanford Encyclopedia of Philosophy, winter 2018 edn, Metaphysics Research Lab. Stanford University. Retrieved May 13, 2021, from https://plato.stanford.edu/archives/win2018/entries/scientific-reproducibility/

Fisher, R. (1947). The design of experiments (4th ed.). Oliver and Boyd.

Gelman, A. (2015). Working through some issues. Significance, 12(3), 33-35. https://doi.org/10.1111/j. 1740-9713.2015.00828.x

Gilboa, I., \& Schmeidler, D. (1989). Maxmin expected utility with non-unique prior. Journal of Mathematical Economics, 18(2), 141-153. https://doi.org/10.1016/0304-4068(89)90018-9

Greaves, H., \& Wallace, D. (2006). Justifying conditionalization: Conditionalization maximizes expected epistemic utility. Mind, 115(459), 607-632. https://doi.org/10.1093/mind/fzl607

Haenni, R., Romeijn, J. W., Wheeler, G., \& Williamson, J. (2011). Probabilistic logics and probabilistic networks. Springer. https://doi.org/10.1007/978-94-007-0008-6

Howson, C., \& Urbach, P. (2006). Scientific reasoning: The Bayesian approach. Open Court Publishing.

Ioannidis, J. P. A. (2005). Why most published research findings are false. PLoS Medicine, 2(8), e124. https://doi.org/10.1371/journal.pmed.0020124

Jaynes, E. T. (1957). Information theory and statistical mechanics. Physical Review, 106(4), 620-630. https://doi.org/10.1103/physrev.106.620

Keynes, J. M. (1921). A treatise on probability. Macmillan and Co.

Kyburg, H. E. (1990). Science and reason. Oxford University Press.

Kyburg, H. E. (2001). Probability as a guide in life. The Monist, 84(2), 135-152. https://doi.org/10.5840/ monist 200184210

Kyburg, H. E. (2003). Are there degrees of belief? Journal of Applied Logic, 1(3-4), 139-149. https://doi. org/10.1016/s1570-8683(03)00010-7

Kyburg, H. E., \& Teng, C. M. (1999). Choosing among interpretations of probability. In Proceedings of the fifteenth conference on uncertainty in artificial intelligence, Morgan Kaufmann, San Francisco CA, USA, UAI'99 (pp. 359-365). https://doi.org/10.5555/2073796.2073837

Kyburg, H. E., \& Teng, C. M. (2001). Uncertain inference. Cambridge University Press. https://doi.org/10. $1017 /$ cbo9780511612947

Levi, I. (1974). On indeterminate probabilities. The Journal of Philosophy, 71(13), 391. https://doi.org/10. $2307 / 2025161$

Mayo, D. G. (1996). Error and the growth of experimental knowledge. The University of Chicago Press.

Mayo, D. G. (2018). Statistical inference as severe testing. How to get beyond the Statistics Wars. Cambridge University Press. https://doi.org/10.1017/9781107286184

McNeish, D. (2016). On using Bayesian methods to address small sample problems. Structural Equation Modeling: A Multidisciplinary Journal, 23(5), 750-773. https://doi.org/10.1080/10705511.2016. 1186549

Mun, J. (2008). Advanced analytical models: Over 800 models and 300 applications from the Basel II Accord to Wall Street and Beyond, Wiley finance series (vol. 419). Wiley. https://doi.org/10.1002/ 9781119197096

Neyman, J. (1941). Fiducial argument and the theory of confidence intervals. Biometrika, 32(2), 128-150. https://doi.org/10.1093/biomet/32.2.128

Neyman, J. (1949). First course in probability and statistics. University of California Press. 
Neyman, J. (1957). "Inductive Behavior" as a basic concept of philosophy of science. Revue de l'Institut International de Statistique/Review of the International Statistical Institute, 25(1/3), 7. https://doi.org/ $10.2307 / 1401671$

Open Science Collaboration. (2015). Estimating the reproducibility of psychological science. Science, 349(6251):aac4716. https://doi.org/10.1126/science.aac4716

Popper, K. R. (1959). The propensity interpretation of probability. The British Journal for the Philosophy of Science, 10(37), 25-42. https://doi.org/10.1093/bjps/x.37.25

Popper, K. R. (2002). The logic of scientific discovery. Routledge.

Rédei, M., \& Gyenis, Z. (2019). Having a look at the Bayes blind spot. Synthese. https://doi.org/10.1007/ s11229-019-02311-9

Reichenbach, H. (1938). Experience and prediction. The University of Chicago Press.

Reichenbach, H. (1971). The theory of probability. University of California Press.

Resnik, M. D. (1987). Choices: An introduction to decision theory. University of Minnesota Press.

Romero, F., \& Sprenger, J. (2020). Scientific self-correction: The Bayesian way. Synthese. https://doi.org/ 10.1007/s11229-020-02697-x

Salmon, W. (1967). The foundations of scientific inference. University of Pittsburgh Press.

Schoenfield, M. (2020). Can imprecise probabilities be practically motivated? A challenge to the desirability of ambiguity aversion. Philosophers Imprint, 20(30), 1-21. http://hdl.handle.net/2027/spo.3521354. 0020.030

Schulte, O. (2018). Formal learning theory. In E. N. Zalta (Ed.), The Stanford Encyclopedia of Philosophy, spring 2018 edn. Metaphysics Research Lab, Stanford University. Retrieved May 21, 2021, from https://web.archive.org/web/20210521152126/https://plato.stanford.edu/entries/learning-formal/

Seabold, S., \& Perktold, J. (2010). Statsmodels: Econometric and statistical modeling with python. In S. van der Walt, \& J. Millman (Eds.), Proceedings of the 9th python in science conference (pp. 92-96). https://doi.org/10.25080/majora-92bf1922-011

Seidenfeld, T. (2004). A Contrast Between two Decision Rules for use with (Convex) Sets of Probabilities: $\Gamma$-Maximin Versus E-Admissibilty. Synthese, 140(1/2), 69-88. https://doi.org/10.1023/b:synt. $0000029942.11359 .8 \mathrm{~d}$

Singh, A. C., Stukel, D. M., \& Pfeffermann, D. (1998). Bayesian versus frequentist measures of error in small area estimation. Journal of the Royal Statistical Society: Series B (Statistical Methodology), 60(2), 377-396. https://doi.org/10.1111/1467-9868.00131

Smaldino, P. E., \& McElreath, R. (2016). The natural selection of bad science. Royal Society Open Science, 3(9), 160384. https://doi.org/10.1098/rsos.160384

Smid, S. C., McNeish, D., Miočević, M., \& van de Schoot, R. (2019). Bayesian versus frequentist estimation for structural equation models in small sample contexts: A systematic review. Structural Equation Modeling: A Multidisciplinary Journal, 27(1), 131-161. https://doi.org/10.1080/10705511.2019.1577140

Spanos, A. (2010). Is frequentist testing vulnerable to the base-rate fallacy? Philosophy of Science, 77(4), 565-583. https://doi.org/10.1086/656009

Sprenger, J., \& Hartmann, S. (2019). Bayesian philosophy of science. Oxford University Press.

Trafimow, D. (2018). An a priori solution to the replication crisis. Philosophical Psychology, 31(8), 11881214. https://doi.org/10.1080/09515089.2018.1490707

Troffaes, M. C. (2007). Decision making under uncertainty using imprecise probabilities. International Journal of Approximate Reasoning, 45(1), 17-29. https://doi.org/10.1016/j.ijar.2006.06.001

van Dongen, N. N. N., van Doorn, J. B., Gronau, Q. F., van Ravenzwaaij, D., Hoekstra, R., Haucke, M. N., Lakens, D., Hennig, C., Morey, R. D., Homer, S., Gelman, A., Sprenger, J., \& Wagenmakers, E. J. (2019). Multiple perspectives on inference for two simple statistical scenarios. The American Statistician, 73(sup1), 328-339. https://doi.org/10.1080/00031305.2019.1565553

Venn, J. (1876). The logic of chance. Macmillan and Co.

von Mises, R. (1957). Probability, statistics and truth. Allen \& Unwin.

Wasserstein, R. L., \& Lazar, N. A. (2016). The ASA statement on p-values: Context, process, and purpose. The American Statistician, 70(2), 129-133. https://doi.org/10.1080/00031305.2016.1154108

Wheeler, G., \& Williamson, J. (2011). Evidential probability and objective Bayesian epistemology. In Philosophy of statistics (pp. 307-331). Elsevier. https://doi.org/10.1016/b978-0-444-51862-0.500095

Williamson, J. (2007). Motivating objective Bayesianism: From empirical constraints to objective probabilities. In W. Harper, \& G. Wheeler (Eds.), Probability and inference: Essays in Honour of Henry E. Kyburg, Jr., Texts in Philosophy (vol. 2, pp. 155-183). College Publications. 
Williamson, J. (2010). In defence of objective Bayesianism. Oxford University Press.

Winsberg, E. (2009). Computer simulation and the philosophy of science. Philosophy Compass, 4(5), 835845. https://doi.org/10.1111/j.1747-9991.2009.00236.x

Publisher's Note Springer Nature remains neutral with regard to jurisdictional claims in published maps and institutional affiliations. 